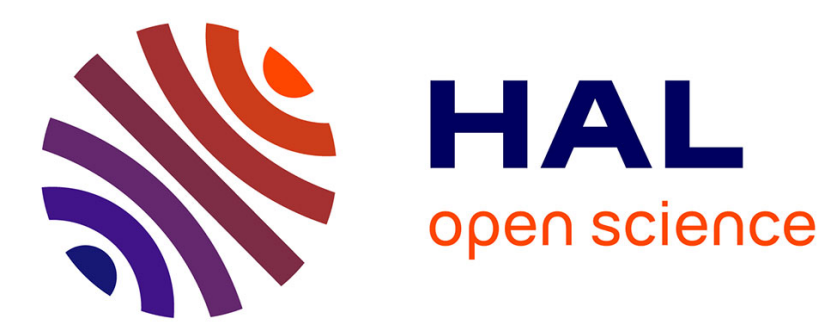

\title{
Improved active control of a functionally graded material beam with piezoelectric patches
}

Isabelle Bruant, Laurent Proslier

\section{To cite this version:}

Isabelle Bruant, Laurent Proslier. Improved active control of a functionally graded material beam with piezoelectric patches. Journal of Vibration and Control, 2013, pp.1077546313506926. 10.1177/1077546313506926 . hal-01421512

\section{HAL Id: hal-01421512 \\ https://hal.parisnanterre.fr/hal-01421512}

Submitted on 17 Jan 2019

HAL is a multi-disciplinary open access archive for the deposit and dissemination of scientific research documents, whether they are published or not. The documents may come from teaching and research institutions in France or abroad, or from public or private research centers.
L'archive ouverte pluridisciplinaire HAL, est destinée au dépôt et à la diffusion de documents scientifiques de niveau recherche, publiés ou non, émanant des établissements d'enseignement et de recherche français ou étrangers, des laboratoires publics ou privés. 


\title{
Improved active control of a functionally graded material beam with piezoelectric patches
}

\author{
I Bruant and L Proslier
}

\begin{abstract}
In this paper, the active vibration control tools are implemented for the vibration control of functionally graded material (FGM) beam with piezoelectric actuators and sensors. The properties of FGM are functionally graded in the thickness direction according to the volume fraction power law distribution. An analytical formulation, based on an efficient trigonometric shear deformation theory, is used to obtain a state space equation. The main steps to set up active control of FGM vibrations are considered in this work. The actuators' and sensors' locations are defined from two optimization problems using controllability and observability gramians. The linear quadratic regulator (LQR) control law, including a state observer is computed. Numerical examples show the influence of the volume fraction index on the observability and controllability properties of the system. The LQR leads to efficient active damping for several kinds of excitations. The study of the uncertainty in the volume fraction index shows the robustness of the control method, and also the possible induced defects.
\end{abstract}

\section{Keywords}

FGM beam, piezoelectric actuators and sensors, active control, optimal location of patches, LQR, Luenberger observer

\section{Introduction}

A new class of composite materials known as "functionally graded materials" (FGMs), first developed by Japanese Scientists in the late 1980s, has attracted much attention these last years. The most common use of this kind of materials is in a mixture of metallic and ceramic characterized by the smooth and continuous change of the mechanical properties from one side to the other side from the thickness. In this way, the ceramic component of FGM is able to withstand a high-temperature environment due to its better thermal resistance while the metal component provides stronger mechanical performance. Due to these superior thermo-mechanical properties, FGMs have found extensive applications in, for example, reactor vessels, fusion energy devices, aircrafts, space vehicles, and other engineering structures, as well as others in the biomedical and defense industries. In this way, much research has been published about vibration and dynamic response of FGM structures like beams and plates. The reader can be referred to Aydogdu and Taskin (2006), Kapuria et al. (2007), Li (2008), Xiang and Yang (2008),
Simsek and Kocaturk (2009), Sina et al. (2009), Atmane et al. (2010), Simsek (2010), Alshorbagy (2011), Doroushi et al. (2011), Giunta et al. (2011), Yao and Shi (2011), Aminbaghai et al. (2012), Jha et al. (2013).

Additionally, in recent years, a great number of research results have been produced about the active vibration control of flexible structures using piezoelectric actuators and sensors. The application of piezoelectric materials has indeed become popular because of their low weight, high strength and easy implementation. The considered systems are usually homogeneous or composite structures (Benjeddou, 2000; Bruant et al., 2008). Less papers deal with the active control

Laboratoire Energétique, Mécanique, Electromagnétisme, Université Paris Ouest Nanterre La De'fense, France

\section{Corresponding author:}

I Bruant, Laboratoire Energétique, Mécanique, Electromagnétisme, EA 44I6, Université Paris Ouest Nanterre La Défense, France. Email: isabelle.bruant@u-paris I0.fr 
of FGMs. Gharib et al. (2008) focused on active vibration control of FGM beams, He et al. (2001), Liew et al. (2003), Kargarnovin et al. (2007), Mirzaeifar et al. (2008), Fakhari and Ohadi (2010), Yiqi and Yiming (2010) and Fu et al. (2013) considered the active control of FGM plates, and Sheng and Wang (2009), Zheng et al. (2009) and Narayanan and Balamurugan (2010) that of the shells.

To simulate the active vibration control of a bending FGM structure, several parameters have to be considered:

1. Concerning the modeling of the FGMs, several displacement field approximations across the thickness can be considered, depending on the dimensions of the structure. For example, in the case of FGM beams, classical theory, first-order and different higher-order shear deformation beam theories can be used (Simsek, 2010).

Elsewhere, different constitutive materials models exist. The most usual is the power law introduced by Xakashima (Aydogdu and Taskin, 2006; Simsek and Kocaturk, 2009) but other models have been developed, such as the exponential law (Aydogdu and Taskin, 2006), or the modified rule of mixtures proposed by Kapuria et al. (2007). In some works, the effective material properties depend continuously on several geometric variables (Xiang and Yang, 2008; Alshorbagy, 2011; Giunta et al., 2011; Aminbaghai et al., 2012).

Finally, depending on the considered structure, analytical development or finite element modeling will be considered.

2. Concerning the active control set up, two criteria have to be defined:

- The locations of piezoelectric patches which have a major influence on the performance of the control system. The study of controllability and observability gramians gives information on the best and worst positions. In addition, in the case of classical elastic materials, sensors and actuators are made with two piezoelectric patches bonded on the top and bottom of the structure. For symmetric structures, this kind of concept is interesting because it induces a possible decoupling between transverse and longitudinal vibrations, and the action of actuators is also more efficient. Actually, in most papers dealing with active control of FGMs, the actuators and sensors are collocated, with actuators on one face and sensors on the other face (He et al., 2001; Kargarnovin et al., 2007; Sheng and Wang, 2009; Yiqi and Yiming, 2010; Fu et al., 2013). Nevertheless, as the top of the FGM should be in a high temperature environment, it is more realistic that a huge piezoelectric patch cannot be located on this face, but only on the bottom face.

- The control law, the most used of which for FGM is the constant gain velocity feedback algorithm (He et al., 2001; Liew et al., 2003; Sheng and Wang, 2009; Zheng et al., 2009; Yiqi and Yiming, 2010; Fu et al., 2013). Nevertheless, in many works about active control of classical structures, linear quadratic regulators are applied (Kondoh et al., 1990; Gaudiller and Der Hagopian, 1996; Preumont, 1999; Bruant et al., 2001; Ang and Wang, 2002; Narayanan and Balamurugan, 2003; Kusculuoglu and Royston, 2005; Bruant et al., 2008; Chomette et al., 2008; Nestorovic and Trajkov, 2010). In Balamurugan and Narayanan (2001), several control laws are compared for active control of plates, and the LQR optimal control schemes are more effective than classical controls. The use of these kinds of control laws requires a state space formulation obtained by a structural modal analysis. Usually, as sensors can only capture a few state variables, a state observer is essential for real-time application, like a Kalman Filter.

To the best of authors' knowledge, no previous work considers all these points for the active control of FGM. Contrary to other investigations, the objectives of this work are to present the main steps for the set up of the active control, and also to show different characteristics related to the properties of FGM. Here, the active control is developed for FGM beams, since this kind of structure plays an important role in structural applications in aerospace and automotive industries and machine elements (Giunta et al., 2011; Aminbaghai et al., 2012). Moreover, the choice of a simple structure like a beam allows us to develop an analytical model.

In Section 2, the active transversal vibration control of a supported FGM beam, equipped with piezoelectric patches located only on its bottom face, is presented. An analytical model of the beam and piezoelectric devices, using an effective trigonometric shear deformation theory, is developed. Section 3 deals with the active control system. First, the actuators and sensors location are defined from an optimization procedure in order to accurately sense and control the first eigenmodes. Then, an LQR algorithm is computed. As this control law needs knowledge of the state of the structure, which is not complete since only the output sensors voltages are observed, a state observer is added to the system control. In Section 4, several numerical tests are presented. In the first simulations, the control vibration suppression is performed for several excitations to show the effectiveness of the control algorithm for the 
FGM beams, even if the actuators are made with only one patch. Then, a sensitivity study on the parameter $k$ (the volume fraction index involved in the FGM constitutive equations) is presented and shows the robustness of the active control law, but also possible consequences related to an error in $k$.

\section{Theory and formulations}

\section{I. Functionally graded beam equipped with piezoelectric patches}

2.I.I. FGM constitutive equations. A functionally graded simply supported beam of length $L$, width $b$, thickness $h$, with co-ordinate system $(O, x, y, z)$, having the origin $O$ is shown in Figure 1. The bottom surface of the FGM is a metal layer and the top surface is a ceramic layer.

$E_{c}, \rho_{c}, E_{m}$ and $\rho_{m}$ denote respectively values of elasticity modulus and mass density of the top and bottom of the beam. It is assumed that the Young's modulus $E$ and mass density $\rho$ vary continuously in the thickness direction ( $z$-axis) according to the power law form:

$$
\begin{gathered}
E(z, k)=\left(E_{c}-E_{m}\right) g(z)+E_{m} \\
\rho(z, k)=\left(\rho_{c}-\rho_{m}\right) g(z)+\rho_{m}
\end{gathered}
$$

where $g(z)=(1 / 2+z / h)^{k}$ is the volume fraction and $k$ is the volume fraction index. It represents the material variation profile through the beam thickness $(0 \leq k \leq \infty)$. Figure 2 shows the variation of $E(z, k)$ through the thickness of an FGM beam, for several values of $k$ (the variation of $\rho$ is similar). The Poisson's ratio is considered constant.

The constitutive equation of the FGM beam is given by the generalized Hooke's law:

$$
\boldsymbol{\sigma}=\mathbf{C} \boldsymbol{\varepsilon}
$$

where $\boldsymbol{\sigma}, \boldsymbol{\varepsilon}$ and $\mathbf{C}$ are, respectively, the stress vector, the strain vector and the usual constitutive matrix for isotropic structure. Its coefficients are functions of $z$, from $E(z, k)$.

2.I.2. Piezoelectric constitutive equation. The beam is equipped with thin piezoelectric patches located on its bottom face. They are used as $N_{a}$ actuators and $N_{s}$ sensors. In order to simplify, it is assumed that the length and thickness of all patches are fixed to $L_{p}$ and $h_{p}$, and the width of piezoelectric is the same as the beam's one. The constitutive relationships describing the electrical and mechanical interactions for piezoelectric materials are given as:

$$
\begin{gathered}
\sigma=\mathbf{c} \varepsilon-\mathbf{e}^{\mathrm{T}} \mathbf{E} \\
\mathbf{D}=\mathbf{e} \boldsymbol{\varepsilon}+\epsilon \mathbf{E}
\end{gathered}
$$

Here $\mathbf{D}$ is the electric displacement vector, $\mathbf{E}=-\operatorname{grad}(\phi)$ is the electric field vector, $\phi$ is the electric potential, $\mathbf{c}$ is the elasticity matrix, $\mathbf{e}$ is the piezoelectric constants matrix and $\epsilon$ is the dielectrical permittivity coefficient matrix. Equation (4) is usually used to model piezoelectric actuators effects on the dynamic of the beam, while equation (5) yields the output equation of sensors (Preumont, 1999).

In order to apply and sense electric potential on piezoelectric actuators and sensors, each patch is covered by electrodes at $z=-\frac{h}{2}$ and $z=-\frac{h}{2}-h_{p}$. Given their small thickness, we can assume that the electric field is constant and only the component $E_{z}$ is nonzero. It induces that:

$$
E_{\mathrm{z}}=-\frac{\Delta \phi}{h_{p}}
$$

where $\Delta \phi$ is the potential difference across the piezoelectric. Finally, the electric displacement satisfies the electrostatic equilibrium equation:

$$
\operatorname{div} \mathbf{D}=0
$$

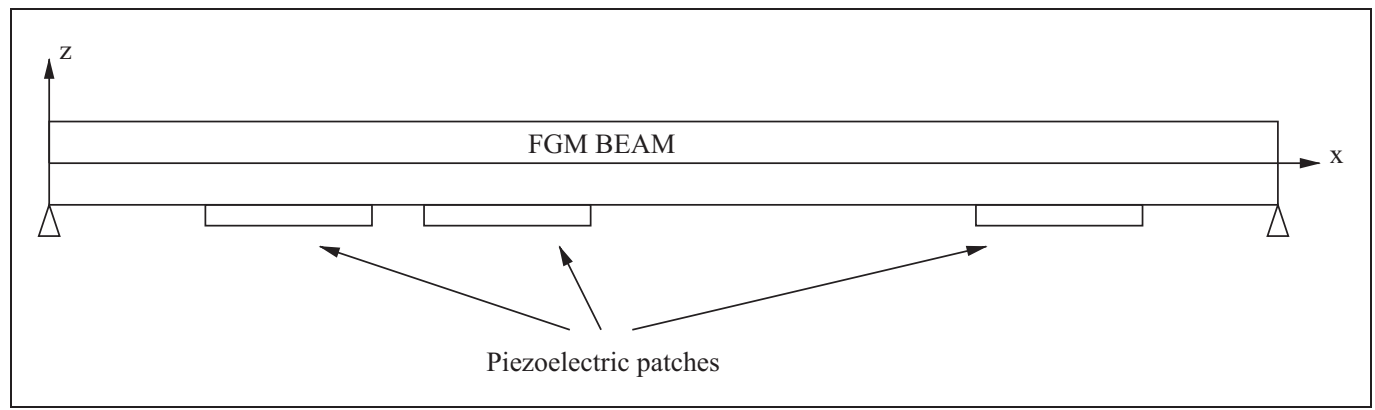

Figure I. A FGM beam equipped with piezoelectric devices. 


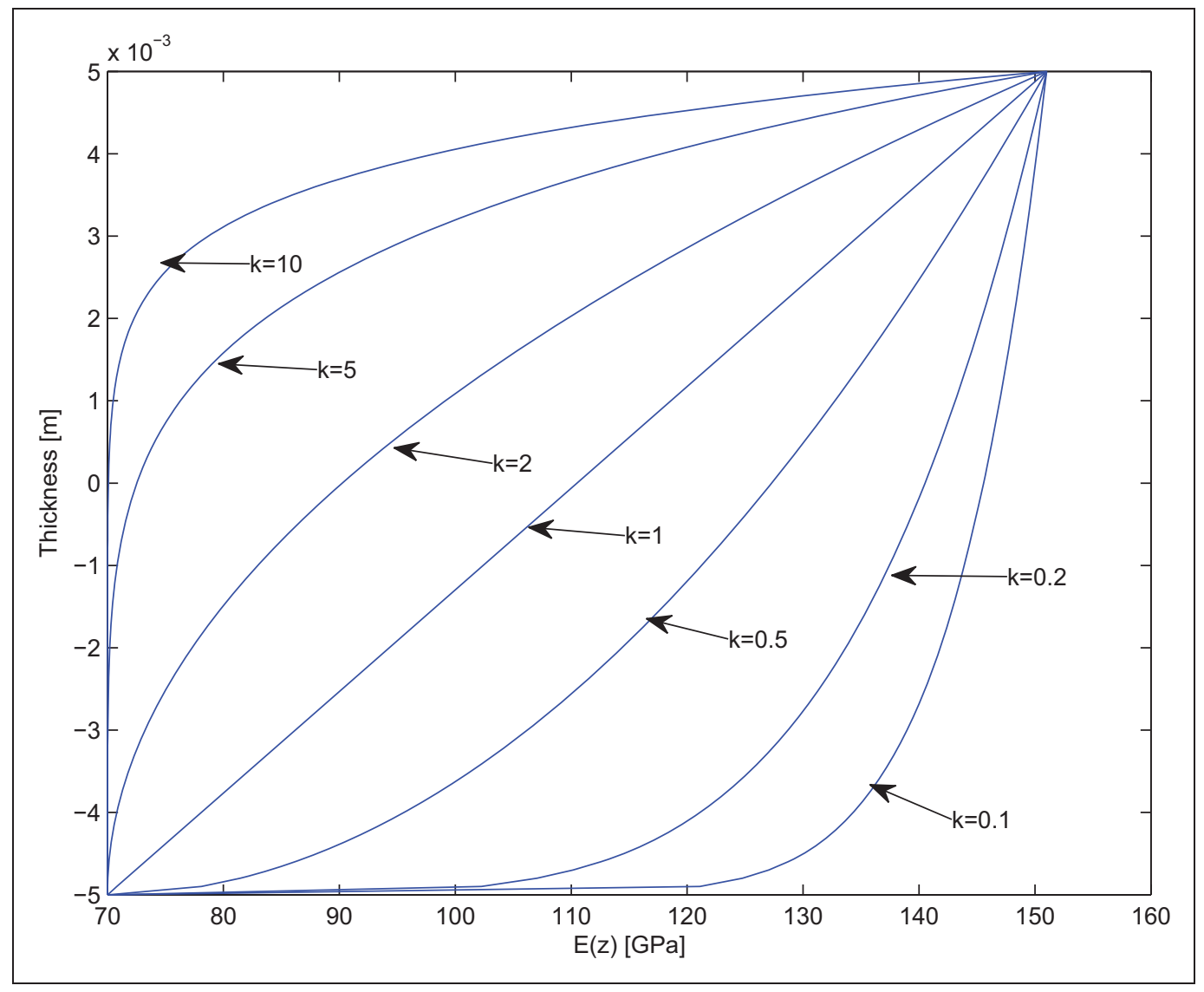

Figure 2. Variation of the Young modulus through the thickness.

which reduces to

$$
\frac{\mathrm{dD}_{z}}{\mathrm{~d} z}=0
$$

\subsection{Strain-displacement relations}

2.2.I. Displacement field approximation. Several deformation beam theories can be used to model FGM beams (Simsek, 2010). Based on the higher-order shear deformation theory, the axial displacement $u_{x}$, and the transverse displacement $u_{z}$ of any point of the beam, are given as:

$$
\begin{aligned}
& u_{x}(x, z, t)=u(x, t)-z v^{\prime}(x, t)+f(z) \gamma(x) \\
& u_{z}(x, z, t)=v(x, t)
\end{aligned}
$$

where $u, v$ and $\gamma$ are respectively the axial, the transverse displacement and transverse shear strain of any point on the neutral axis. $\gamma$ is given by:

$$
\gamma(x)=\theta(x, t)+v^{\prime}(x, t)
$$

$\theta(x, t)$ is the total bending rotation of the cross-sections at any point on the neutral axis. $t$ denotes time and ' indicates the first derivative with respect to $x$.

Different beam theories can be obtained by choosing $f(z)$ as follows:

- Euler Bernoulli theory (or classical beam theory): $f(z)=0$;

- Timoshenko theory (or first order shear deformation beam theory): $f(z)=z$;

- Several higher-order beam theories exist, which consider the warping of the cross-sections and satisfy the zero transverse shear stress condition of the upper and lower fibers of the cross-section without a shear correction factor. One of them is the trigonometric shear deformation beam theory where $f(z)=\frac{h}{\pi} \sin \left(\frac{\pi z}{h}\right)$ (Polit and Touratier, 2000). It is interesting to note that since the sine function has an infinite radius convergence, equation (9) can be written for the inplane displacement as:

$$
u_{x}=u-z v^{\prime}+z \gamma-\frac{\pi^{2}}{3 ! h^{2}} z^{3} \gamma+\frac{\pi^{4}}{5 ! h^{4}} z^{5} \gamma+\cdots
$$


If $\gamma=0$ we obtain the Euler-beam kinematics, if we develop the first order sine function, we obtain the Timoshenko theory, and if we develop the sine function to the third-order only, we then obtain the same order kinematics as in Simsek (2010).

2.2.2. Equations of motion. In order to find the mechanical governing equations of FGM beam, the start point is the $3 \mathrm{D}$ variational principle given by the problem find $\mathbf{u}$ such that $\forall \mathbf{u}^{*}$

$$
\int_{\omega} \rho \mathbf{u}^{*} \cdot \ddot{\mathbf{u}} \mathrm{dv}=-\int_{\omega} \boldsymbol{\epsilon}\left(\mathbf{u}^{*}\right)^{\mathrm{T}} \boldsymbol{\sigma} \mathrm{dv}+\int_{\omega} \mathbf{u}^{*} \cdot \mathbf{f d v}+\int_{\partial \omega_{\mathrm{F}}} \mathbf{u}^{*} \cdot \mathbf{F d S}
$$

where $\omega$ represents the FGM beam with piezoelectric patches. $\mathbf{f}$ and $\mathbf{F}$ are the prescribed body and surface forces applied on $\partial \omega_{\mathrm{F}}$. In this study they will be restricted to the $z$-component: $f_{z}$ and $F_{z}$. Furthermore, $\mathbf{u}^{*}$ is the admissible virtual displacement and $\boldsymbol{\epsilon}\left(\mathbf{u}^{*}\right)$ is the virtual strain.

By substituting the FGM and piezoelectric stressstrain relations into (12), the following constitutive equations can be obtained:

$$
\begin{gathered}
{\left[\begin{array}{c}
N x \\
\bar{M}_{y} \\
M_{y}
\end{array}\right]=\mathbf{K}\left[\begin{array}{c}
u^{\prime} \\
v^{\prime \prime} \\
\theta^{\prime}
\end{array}\right]+\sum_{i=1}^{N_{a}}\left[\begin{array}{c}
\left\langle e_{31}\right\rangle \\
\left\langle e_{31}(f(z)-z)\right\rangle \\
-\left\langle e_{31} f\right\rangle
\end{array}\right] \frac{\left(\Delta \phi_{a}\right)_{i}}{h_{p}}} \\
T_{z}=\left\langle G f^{\prime 2}\right\rangle\left(\theta+v^{\prime}\right)
\end{gathered}
$$

where $\mathbf{K}$ equals

$$
\left[\begin{array}{ccc}
\langle E, 1\rangle & \langle E, f\rangle-\langle E, z\rangle & \langle E, f\rangle \\
\langle E, f\rangle-\langle E, z\rangle & \left\langle E, f^{2}\right\rangle+\left\langle E, z^{2}\right\rangle-2\langle E, f z\rangle & \left\langle E, f^{2}\right\rangle-\langle E, f z\rangle \\
\langle E, f\rangle & \left\langle E, f^{2}\right\rangle-\langle E, f z\rangle & \left\langle E, f^{2}\right\rangle
\end{array}\right]
$$

and $\left(\Delta \phi_{a}\right)_{i}$ is the potential field for the $i$ th actuator. Three equilibrium equations are also deduced:

$$
\begin{gathered}
\langle m, 1\rangle \ddot{u}+(\langle m, f\rangle-\langle m, z\rangle) \ddot{v}^{\prime}+\langle m, f\rangle \ddot{\theta}=N_{x}^{\prime} \\
\langle m, 1\rangle \ddot{v}+(\langle m, z\rangle-\langle m, f\rangle) \ddot{u}^{\prime}+\left(\langle m, z f\rangle-\left\langle m, f^{2}\right\rangle\right) \ddot{\theta}^{\prime}+\cdots \\
+\left(2\langle m, z f\rangle-\left\langle m, z^{2}\right\rangle-\left\langle m, f^{2}\right\rangle\right) \ddot{v}^{\prime \prime}=-\bar{M}_{y}^{\prime \prime}+T_{z}+\left\langle f_{z}\right\rangle
\end{gathered}
$$

$$
\langle m, f\rangle \ddot{u}+\left\langle m, f^{2}\right\rangle \ddot{\theta}+\left(\left\langle m, f^{2}\right\rangle-\langle m, z f\rangle\right) \ddot{v}^{\prime}=M_{y}^{\prime}-T_{z}
$$

All coefficients $\langle\cdot\rangle$ are detailed in Appendix A.
To complete these equations, boundary conditions must be added. In this study, we consider simply supported boundary conditions, given by:

$$
\begin{aligned}
& N_{x}(x=0)=\bar{M}_{y}(x=0)=M_{y}(x=0)=0 \\
& N_{x}(x=L)=\bar{M}_{y}(x=L)=M_{y}(x=L)=0
\end{aligned}
$$

2.2.3. Sensors equation. In the case of the sensors output equation, there exist two basic approaches for calculating the piezoelectric sensor voltage (Jha and Inman, 2003). One approach is to consider an open-circuit configuration in which the total surface charge is assumed to be zero and the sensor voltage is obtained by integrating the electric field over the sensor. Another approach is to consider a closed-circuit configuration in which the electric field becomes zero and the total charge on the sensor is obtained by integrating the electric displacement over the sensor area. Here, the first approach is followed.

From (8) we get for the $i$ th sensor (Kargarnovin et al., 2007):

$$
\frac{1}{2} \int_{S} D_{z}(z=-h / 2)+D_{z}\left(z=-h / 2-h_{p}\right) \mathrm{d} x \mathrm{~d} y=0
$$

where $S$ is the effective electrode surface, assumed equal to $S=L_{p} \times l$. By substituting the electric displacement by the relation (5), the electric voltage of the $i$ th sensor, located at $c_{i}$, is given by:

$$
\begin{aligned}
\left(\Delta \phi_{s}\right)_{i}= & \frac{h_{p} e_{31}}{L_{p} \epsilon_{33}}\left\{u\left(c_{i}+L_{p}\right)-u\left(c_{i}\right)+\frac{1}{2}\left(f\left(-h / 2-h_{p}\right)\right.\right. \\
& \left.+f\left(-h_{p}\right)+\left(h+h_{p}\right)\right)\left(v^{\prime}\left(c_{i}+L_{p}\right)-v^{\prime}\left(c_{i}\right)\right) \\
& \left.+\frac{1}{2}\left(f\left(-h / 2-h_{p}\right)+f\left(-h_{p}\right)\right)\left(\theta\left(c_{i}+L_{p}\right)-\theta\left(c_{i}\right)\right)\right\}
\end{aligned}
$$

Again, as the piezoelectric sensor is only on the bottom face of the beam, there is no possible decoupling between $u$ and $v$.

In order to set up a control law, damping the vibrations caused by external disturbances or by initial conditions, a state space model is developed in the next section and a linear quadratic regulator (LQR) method, including a state observer is used.

\section{The control system}

\section{I. Modal analysis}

The application of the active control methods in a dynamic structural problem requires the use of a state 
space model. To obtain this kind of equation, the solution vector $\mathbf{U}$ is decomposed into a normalized orthogonal structural modal basis. Eigenmodes and eigenfrequencies are obtained by solving the free vibration problem where:

- the external load vectors and electric potential actuators are taken as zero:

$$
\mathbf{f}=\mathbf{F}=\left(\Delta \phi_{a}\right)_{i}=0 \quad \forall i=1, \ldots, N_{a}
$$

- the time dependent displacement can be expressed as follows:

$$
\mathbf{U}=\mathrm{e}^{\mathrm{i} \omega t} \overline{\mathbf{U}}
$$

where $\omega$ is the natural frequency of the beam.

To solve free vibration equations (13), (16), (17), (18), (22) and (23), that satisfy the boundary conditions (19), we suppose that (Reddy, 2004):

$$
\begin{aligned}
p=1,2, \ldots & \\
u(x) & =u_{0} \cos \frac{p \pi x}{L} \\
v(x) & =v_{0} \sin \frac{p \pi x}{L} \\
\theta(x) & =\theta_{0} \cos \frac{p \pi x}{L}
\end{aligned}
$$

It leads to the following eigenvalue problem: find $\left(\omega, \mathbf{U}_{\mathbf{0}}=\left(u_{0}, v_{0}, \theta_{0}\right)^{\mathrm{T}}\right)$ which satisfy:

$$
\omega^{2} \mathbf{M}_{\mathbf{0}} \mathbf{U}_{\mathbf{0}}=\mathbf{K}_{\mathbf{0}} \mathbf{U}_{\mathbf{0}}
$$

$\mathbf{M}_{\mathbf{0}}$ and $\mathbf{K}_{\mathbf{0}}$ are respectively the inertia and stiffness matrix. They depend on $p$ and are given in Appendix B. The solution of (27) gives the $N$ first eigenfrequencies $\omega_{1}<\omega_{2}<\cdots<\omega_{N}$, and the eigenmodes are $\mathbf{U}_{\mathbf{n}}$ which components are defined by: $n=1, \ldots, N$

$$
\begin{aligned}
& u_{n}(x)=\left(u_{0}\right)_{n} \cos \frac{p_{n} \pi x}{L} \\
& v_{n}(x)=\left(v_{0}\right)_{n} \sin \frac{p_{n} \pi x}{L} \\
& \theta_{n}(x)=\left(\theta_{0}\right)_{n} \cos \frac{p_{n} \pi x}{L}
\end{aligned}
$$

$p_{n}$ is the value of the associated coefficient $p$. The eigenmodes are normalized with respect to the mass.

In this way, assuming that the system response is governed by the $N$ first eigenmodes, the displacement can be expressed by:

$$
\mathbf{U}=\sum_{n=1}^{N} \alpha_{n}(t) \mathbf{U}_{\mathbf{n}}
$$

Substituting this equation into the equations of the problem and (12), and using the orthogonality properties of modes lead to the following equations:

$\ddot{\alpha}_{n}+2 \delta \omega_{n} \dot{\alpha}_{n}+\omega_{n}^{2} \alpha_{n}=l\left(\mathbf{U}_{\mathbf{n}}\right)+\sum_{i=1}^{N_{a}} b_{n i}\left(\Delta \phi_{a}\right)_{i} \quad \forall n=1, \ldots, N$

where

$$
\begin{gathered}
l\left(\mathbf{U}_{\mathbf{n}}\right)=\int_{0}^{L}\left\langle f_{z}\right\rangle v_{n}^{\prime} \mathrm{d} x \\
b_{n i}=-\frac{1}{h_{p}} \int_{a_{i}}^{a_{i}+L_{p}} u_{n}^{\prime}(x)\left\langle e_{31} S\right\rangle+\theta_{n}^{\prime}(x)\left\langle e_{31} f\right\rangle \\
+v_{n}^{\prime \prime}(x)\left\langle e_{31}(f(z)-z)\right\rangle \mathrm{d} x
\end{gathered}
$$

and $a_{i}$ is the location of the $i$ th actuator. A term of modal viscous damping has been added to take into account a small amount of natural damping without coupling the modes. $\delta$ is the damping ratio.

If the actuators are made with two patches located symmetrically at the top and bottom of the beam, it is possible to make $u_{n}$ disappear in (34) imposing the two patches in phase opposition. Unfortunately, in the case of FGM structure, as the piezoelectric actuators are only on the bottom face of the beam, there is no possible decoupling between axial and transversal displacement.

Additionally, from (21), the ith sensor's output equation becomes:

$$
\left(\Delta \phi_{s}\right)_{i}=\sum_{n=1}^{N} \alpha_{n}(t) d_{i n}
$$

where

$$
\begin{aligned}
d_{i n}= & \frac{h_{p} e_{31}}{L_{p} \epsilon_{33}}\left\{u_{n}\left(c_{i}+L_{p}\right)-u_{n}\left(c_{i}\right)+\frac{1}{2}\left(f\left(-h / 2-h_{p}\right)\right.\right. \\
& \left.+f\left(-h_{p}\right)+\left(h+h_{p}\right)\right)\left(v_{n}^{\prime}\left(c_{i}+L_{p}\right)-v_{n}^{\prime}\left(c_{i}\right)\right) \\
& \left.+\frac{1}{2}\left(f\left(-h / 2-h_{p}\right)+f\left(-h_{p}\right)\right)\left(\theta_{n}\left(c_{i}+L_{p}\right)-\theta_{n}\left(c_{i}\right)\right)\right\}
\end{aligned}
$$


These equations can be written in a state space form. Using the state vector (size $2 N$ )

$$
\mathbf{x}=\left\{\omega_{n} \alpha_{n}, \quad \dot{\alpha}_{n}\right\}^{\mathrm{T}}
$$

yields

$$
\begin{gathered}
\dot{\mathbf{x}}=\mathbf{A} \mathbf{x}+\mathbf{B} \Delta \phi_{\mathbf{a}}+\mathbf{G} \\
\mathbf{x}(t=0)=\mathbf{x}_{\mathbf{0}}, \quad \mathbf{y}=\Delta \phi_{\mathbf{s}}=\mathbf{C} \mathbf{x}
\end{gathered}
$$

$\mathbf{A}_{2 N, 2 N}, \mathbf{B}_{2 N, N_{a}}, \mathbf{C}_{N_{s}, 2 N}$ and $\mathbf{G}_{2 N, 1}$ are the state, control, output and load matrices, given by:

$$
\begin{gathered}
\mathbf{A}=\left[\begin{array}{cc}
\mathbf{0} & \boldsymbol{\omega} \\
-\boldsymbol{\omega} & -2 \delta \boldsymbol{\omega}
\end{array}\right] \quad \mathbf{B}=\left[\begin{array}{ll}
\mathbf{0} & \mathbf{b}
\end{array}\right]^{\mathrm{T}} \\
\mathbf{C}=\left[\begin{array}{ll}
\mathbf{d} \omega^{-1} & \mathbf{0}
\end{array}\right] \quad \mathbf{G}=\left[\begin{array}{l}
\mathbf{0} \\
\mathbf{g}
\end{array}\right]
\end{gathered}
$$

$\mathbf{x}_{\mathbf{0}}$ is the initial conditions vector, $g_{n}=l\left(\mathbf{U}_{\mathbf{n}}\right)$, and $\boldsymbol{\omega}$ is the $N \times N$ diagonal matrix with $\omega_{n n}=\omega_{n}$.

\subsection{Actuators and sensors location}

The actuators and sensors location have a major influence on the performance of the control system. Misplaced sensors and actuators lead to problems such as the lack of observability or controllability. For this reason, many studies have been published on this subject and different cost functions have been used to find the optimal locations of these active elements. In this paper, the optimal location of sensors and actuators are computed independently. The modified optimization criteria developed in Bruant et al. (2010) are used. They ensure good observability and good controllability of each mode, considering them with homogeneity, and not globally as it is usually the case. We briefly give the method in the two next subsections.

3.2.I. Actuators location. The objective here is to find actuators locations that minimize the control energy required to bring the modal system to a desired state $\mathbf{x}_{\mathbf{T}}$ after some time $T$ :

$$
J=\min _{\Delta \phi_{a}} \int_{0}^{T}\left\{\boldsymbol{\Delta} \boldsymbol{\phi}_{a}^{T} \boldsymbol{\Delta} \boldsymbol{\phi}_{a}\right\} \mathrm{d} t
$$

The optimal solution gives the following optimal control energy:

$$
J=\left(\mathbf{e}^{\mathbf{A}^{\mathbf{T}}} \mathbf{x}_{\mathbf{0}}-\mathbf{x}_{\mathbf{T}}\right)^{T}(\mathbf{W}(\mathbf{T}))^{-1}\left(\mathbf{e}^{\mathbf{A}^{\mathrm{T}}} \mathbf{x}_{\mathbf{0}}-\mathbf{x}_{\mathbf{T}}\right)
$$

where $\mathbf{W}(\mathbf{T})$ is the controllability gramian matrix defined by:

$$
\mathbf{W}(\mathbf{T})=\int_{0}^{T} \mathbf{e}^{\mathbf{A t}} \mathbf{B B}^{\mathbf{T}} \mathbf{e}^{\mathbf{A}^{\mathrm{T}} \mathbf{t}} \mathrm{d} t
$$

Minimizing $J$ with respect to the actuators locations consists in minimizing $(\mathbf{W}(\mathbf{T}))^{-\mathbf{1}}$ or maximizing a measure of the controllability gramian matrix (Hac and Liu, 1993). Instead of using $\mathbf{W}(\mathbf{T})$, a steady state $\mathbf{W}_{\mathbf{c}}$ can be considered to eliminate the dependency of the solution $T$. $\mathbf{W}_{\mathbf{c}}$ tends to a diagonal form with

$$
\left(W_{c}\right)_{n n}=\left(W_{c}\right)_{n+N, n+N}=\frac{1}{4 \delta \omega_{n}} \sum_{j=1}^{N_{a}} b_{n j}^{2}
$$

$\left(W_{c}\right)_{n n}$ equals the energy transmitted from the actuators to the structure for the $n$th eigenmode. Hence, if the eigenvalue $\left(W_{c}\right)_{n n}$ is small, the $n$th eigenmode is difficult to control: there is no controllability for the system.

The usual criteria take into account globally the eigenmode. Instead of maximizing a global norm of $\mathbf{W}_{\mathbf{c}}$ which means minimizing the electrical energy, the optimization problem considered here is: to find the actuators' location which maximizes

$$
\begin{aligned}
J_{A} & =\min _{n=1, N} \frac{\left(W_{c}\left(a_{1}, \ldots, a_{N_{a}}\right)\right)_{n n}}{\max _{a_{1}, \ldots, a_{N_{a}}}\left(W_{c}\left(a_{1}, \ldots, a_{N_{a}}\right)\right)_{n n}} \\
& =\min _{n=1, N} \frac{\sum_{j=1}^{N_{a}} b_{n j}^{2}}{\max _{a_{1}, \ldots, a_{N_{a}}} \sum_{j=1}^{N_{a}} b_{n j}^{2}}
\end{aligned}
$$

and,

$$
\forall n=1, \ldots, N \quad 0 \leq \frac{\left(W_{c}\left(a_{1}, \ldots, a_{N_{a}}\right)\right)_{i i}}{\max _{a_{1}, \ldots, a_{N_{a}}}\left(W_{c}\left(a_{1}, \ldots, a_{N_{a}}\right)\right)_{i i}} \leq 1
$$

The greatest advantage of this criterion is that all modes are studied with the same range. Furthermore, the expression inside (46) has a physical meaning: it is the mechanical energy transmitted for the $n$th mode divided by the maximal mechanical energy that could be received.

3.2.2. Sensors' location. The optimal location of sensors is determined in the same way as the optimal location of actuators. It consists in maximizing the gramian observability matrix defined by:

$$
\mathbf{W}_{\mathbf{o}}=\int_{0}^{\infty} \mathbf{e}^{\mathbf{A}^{\mathrm{T}} \mathbf{t}} \mathbf{C}^{\mathrm{T}} \mathbf{C} \mathbf{e}^{\mathrm{At}} \mathrm{dt}
$$


which is diagonal dominant:

$$
\left(W_{o}\right)_{n n}=\left(W_{o}\right)_{n+N, n+N}=\frac{1}{4 \delta \omega_{n}} \sum_{j=1}^{N_{s}} c_{j n}^{2} \quad n=1, \ldots, N
$$

To have convenient information about the $N$ first eigenmodes, and to insure homogeneity between each term $\left(W_{o}\right)_{i i}$, the optimization problem considered here is: to find the sensors locations $c_{1}, \ldots, c_{N_{s}}$ which maximize

$$
\begin{aligned}
J_{S} & =\min _{n=1, N} \frac{\left(W_{o}\left(c_{1}, \ldots, c_{N_{s}}\right)\right)_{n n}}{\max _{c_{1}, \ldots, c_{N_{s}}}\left(W_{o}\left(c_{1}, \ldots, c_{N_{s}}\right)\right)_{n n}} \\
& =\min _{n=1, N} \frac{\sum_{j=1}^{N_{s}} c_{j n}^{2}}{\max _{c_{1}, \ldots, c_{N_{s}}} \sum_{j=1}^{N_{s}} c_{j n}^{2}}
\end{aligned}
$$

with $\forall n=1, \ldots, N \quad 0 \leq \frac{\left(W_{o}\left(c_{1}, \ldots, c_{N_{s}}\right)\right)_{n n}}{\max _{c_{1}, \ldots, c_{N_{s}}}\left(W_{o}\left(c_{1}, \ldots, c_{N_{s}}\right)\right)_{n n}} \leq 1$

$\max _{c_{1}, \ldots, c_{N_{s}}}\left(W_{o}\left(c_{1}, \ldots, c_{N_{s}}\right)\right)_{n n}$ represents the maximal output energy which could be measured for the $n$th mode by the sensors.

3.2.3. Application to the simply supported FGM beam. In this study, the criteria (46) and (49) become:

$J_{A}=\min _{n=1, N} \frac{\sum_{j=1}^{N_{s}}\left[\cos \left(\frac{p_{n} \pi}{L}\left(a_{j}+L_{p}\right)\right)-\cos \left(\frac{p_{n} \pi}{L} a_{j}\right)\right]^{2}}{\max _{a_{1}, \ldots, a_{N s}} \sum_{j=1}^{N_{s}}\left[\cos \left(\frac{p_{n} \pi}{L}\left(a_{j}+L_{p}\right)\right)-\cos \left(\frac{p_{n} \pi}{L} a_{j}\right)\right]^{2}}$

$J_{S}=\min _{n=1, N} \frac{\sum_{j=1}^{N_{s}}\left[\cos \left(\frac{p_{n} \pi}{L}\left(c_{j}+L_{p}\right)\right)-\cos \left(\frac{p_{n} \pi}{L} c_{j}\right)\right]^{2}}{\max _{c_{1}, \ldots, c_{N_{s}}} \sum_{j=1}^{N_{s}}\left[\cos \left(\frac{p_{n} \pi}{L}\left(c_{j}+L_{p}\right)\right)-\cos \left(\frac{p_{n} \pi}{L} c_{j}\right)\right]^{2}}$

Normalizing each diagonal term of gramian matrix, these two criteria are independent on $k$ and $\frac{h}{L}$, and finally, are similar for all kinematics. Moreover, in the case where all patches have the same dimensions and $N_{a}=N_{s}$, actuators and sensors have the same optimal locations.

\subsection{Control law and observer}

In order to actively control vibrations, rather than what is done in most papers about FGM vibrations, the efficient linear quadratic regulator is used (Kailath, 1980). Assuming that the state equation is controllable, the control law may be written as:

$$
\Delta \phi_{a}=-\mathbf{K x}
$$

which minimizes a cost function given by:

$$
J_{\phi}=\frac{1}{2} \int_{0}^{\infty}\left(\mathbf{x}^{\mathrm{T}} \mathbf{Q} \mathbf{x}+\Delta \phi_{\mathbf{a}}^{\mathrm{T}} \mathbf{R} \Delta \phi_{\mathrm{a}}\right) \mathrm{d} t
$$

$\mathbf{R}$ is a positive matrix and $\mathbf{Q}$ is a positive semidefinite matrix. The optimal solution is

$$
\mathbf{K}=\mathbf{R}^{-\mathbf{1}} \mathbf{B}^{\mathbf{T}} \mathbf{P}
$$

where $\mathbf{P}$ satisfies the Riccati equation:

$$
\mathbf{A}^{\mathrm{T}} \mathbf{P}+\mathbf{P A}-\mathbf{P B R}^{-1} \mathbf{B}^{\mathrm{T}} \mathbf{P}+\mathbf{Q}=\mathbf{0}
$$

The choice of $\mathbf{Q}$ and $\mathbf{R}$ is not easy (Ang and Wang, 2002). In the following applications, $\mathbf{Q}$ is chosen so that $\mathbf{x}^{\mathbf{T}} \mathbf{Q} \mathbf{x}$ represents the mechanical energy. The components of $\mathbf{R}$ are chosen using the following statement: the maximal values of $\boldsymbol{\Delta} \boldsymbol{\phi}_{a}$ are less than the maximal admissible values of the piezoelectric materials.

It is important to note that in order to be implemented, the optimal state control law needs knowledge of the state vector $\mathbf{x}$. This knowledge is not complete since only the output voltages in $\mathbf{y}$ are observed. Assuming that the state system verifies the observability criteria, an estimation $\hat{\mathbf{x}}$ is computed using a Luenberger observer (Kailath, 1980) which is thus:

$$
\frac{\mathrm{d}}{\mathrm{d} t} \hat{\mathbf{x}}=\mathbf{A} \hat{\mathbf{x}}+\mathbf{B} \Delta \phi_{\mathrm{a}}+\mathbf{L}(\mathbf{y}-\mathbf{C} \widehat{\mathbf{x}})+\mathbf{G}
$$

where $\mathbf{L}$ is the observance gain matrix. It is chosen so that the real part of the eigenvalues of $\mathbf{A}-\mathbf{L C}$ are negative. Consequently, the control law applied to the actuators becomes:

$$
\Delta \phi_{a}=-\mathbf{K} \hat{\mathbf{x}}
$$

\section{Numerical examples}

In the applications, the FGM beam is equipped with actuator and sensor respectively made of PZT5A and PVDF piezoelectric patch. They are assumed to be perfectly bonded to the bottom surface of the beam, and their thickness is assumed to be small compared to the beam thickness. Then, the rigidity and mass of the patches are neglected in the mechanical problem. The geometrical and mechanical properties of the system are detailed in Tables 1-3. In all simulations, the first six frequencies are considered and correspond to transversal eigenmodes. The objective is to control the first three eigenmodes. 
Table I. Geometrical characteristics of the beam and the piezoelectric patch.

\begin{tabular}{llll}
\hline & Beam & $\begin{array}{l}\text { Piezoelectric } \\
\text { sensor }\end{array}$ & $\begin{array}{l}\text { Piezoelectric } \\
\text { actuator }\end{array}$ \\
\hline Length $(\mathrm{m})$ & 0.7 & 0.06 & 0.06 \\
Width $(\mathrm{m})$ & 0.02 & 0.02 & 0.02 \\
Thickness $(\mathrm{m})$ &. & 0.0005 & 0.001 \\
\hline
\end{tabular}

Table 2. Characteristics of piezoelectric patch PVDF and PZT5A.

\begin{tabular}{lll}
\hline & $\begin{array}{l}\text { Piezoelectric } \\
\text { sensor (PVDF) }\end{array}$ & $\begin{array}{l}\text { Piezoelectric } \\
\text { actuator (PZT5A) }\end{array}$ \\
\hline$\epsilon_{33}\left(\mathrm{~F} \mathrm{~m}^{-1}\right)$ & $1.062 \times 10^{-8}$ & $1.5 \times 10^{-8}$ \\
$\mathrm{e}_{31}\left(\mathrm{C} \mathrm{m}^{-2}\right)$ & 0.046 & -7.209 \\
\hline
\end{tabular}

Table 3. Mechanical characteristics of the FGM beam.

\begin{tabular}{lll}
\hline & Zirconia (ceramic) & Aluminium (metal) \\
\hline$\rho\left(\mathrm{kg} \mathrm{m}^{-3}\right)$ & 3000 & 2700 \\
$E(\mathrm{GPa})$ & $15 \mathrm{I}$ & 70 \\
$v$ & 0.3 & 0.3 \\
$\delta$ & $\mathrm{I} \times 10^{-4}$ & \\
\hline
\end{tabular}

\section{I. Choice of the kinematics}

Depending on the ratio $\frac{L}{h}$, a simple or a higher-order beam theory must be used. It is illustrated in Figures 3 and 4 , where the variation with $k$ of the following difference between the frequency obtained by Euler Bernoulli model $f_{\mathrm{EB}}(n)$, and the frequency obtained by the Sinus model $f_{\mathrm{SIN}}(n)$ :

$$
\operatorname{Dif}(n)=\left|\frac{f_{\mathrm{EB}}(n)-f_{\mathrm{SIN}}(n)}{f_{\mathrm{SIN}}(n)}\right| 100 \quad n=1, \ldots, N
$$

is plotted for the ratios $\frac{L}{h}=10$ and $\frac{L}{h}=35$.

For the first ratio, the difference between results with the Euler Bernoulli and Sinus theories are very important, except for the first frequency. The use of the Sinus model is essential for this kind of ratio. In the following tests, the ratio $\frac{L}{h}=35$ will be used. For this ratio, results are quite similar. Nevertheless, as the difference for the last frequency can be higher than $4 \%$, we will use this kinematics in this paper.

\section{2. $\mathrm{k}$ influence on the controllability and observability index}

The efficiency of the control system can be evaluated by the two gramian matrices previously defined in (44) and (47). The higher diagonal values, the better the controllability or observability of the system. The variation of their first normalized diagonal values with $k$ are shown Figures 5 and 6 (the curves for the others eigenvalues are similar). It appears that, for high a $k$ value, the controllability and observability are maximal. This is due to the metallic part of the FGM which is predominant in this case: as the beam is more flexible, it is easier to sense and to actuate to its vibration. Nevertheless, a peak exists for the controllability curve. It comes from the coupling between axial and transversal displacement in the component $b_{11}$ (34). As the axial displacement $u$ is in the expression of $b_{11}$, it has an effect on the variation of $\left(W_{c}\right)_{11}$ with $k$.

\subsection{Active control of the FGM beam}

The previous simulations show the influence of the parameter $k$ on the controllability and observability of the system. In this section, we present three tests of active control of the beam, using the LQR method. The parameter $k$ is fixed to 5 . The construction of the control law and observer is done using MATLAB. The objective is to control the three first eigenfrequencies (for $k=5: f_{1}=106 \mathrm{~Hz}, f_{2}=420 \mathrm{~Hz}$ and $f_{3}=939 \mathrm{~Hz}$ ). Accordingly, in all tests, the beam is equipped with only one sensor. Its best location, obtained with optimal criteria (51), is $c_{1}=0.495 \mathrm{~m}$. The electrical potential is set to zero on all the interface between FGM and piezoelectric patches.

4.3.I. Release test with two actuators. First, we study the active control of the FGM beam in the case of a release test. Only the two first eigenmodes are excited from an initial load $f_{z}(x)=5.10^{4}(\cos (\pi x / L)+\cos (\pi 2 x / L))$. In this test, two actuators are used and located, from optimal criteria (50), at $0.145 \mathrm{~m}$ and $0.4367 \mathrm{~m}$. $\mathbf{R}$ is considered diagonal and its values are chosen in order not to exceed the maximal admissible value of electric potential. The observer estimates the state of the system in $0.1 \mathrm{~s}$.

The sensor output in the open and closed loop is plotted in Figure 7. The amplitude decay of the open loop response comes from natural damping, while that of the closed loop system comes mainly from the feedback control. Figures 8 and 9 show the required input actuators' voltages for the active control. The vibrations vanish in less than $7 \mathrm{~s}$. If the components of $\mathbf{R}$ are chosen to be smaller, the maximal values of the input voltages will be higher and the control will be more efficient.

4.3.2. Step load test with one actuator. The structure is now subjected to a load step $5.10^{4} \mathrm{~N}$ at the location $[L / 3, L / 3+L / 4]$, for $2.5 \mathrm{~s}$ (Figure 10). It is controlled 


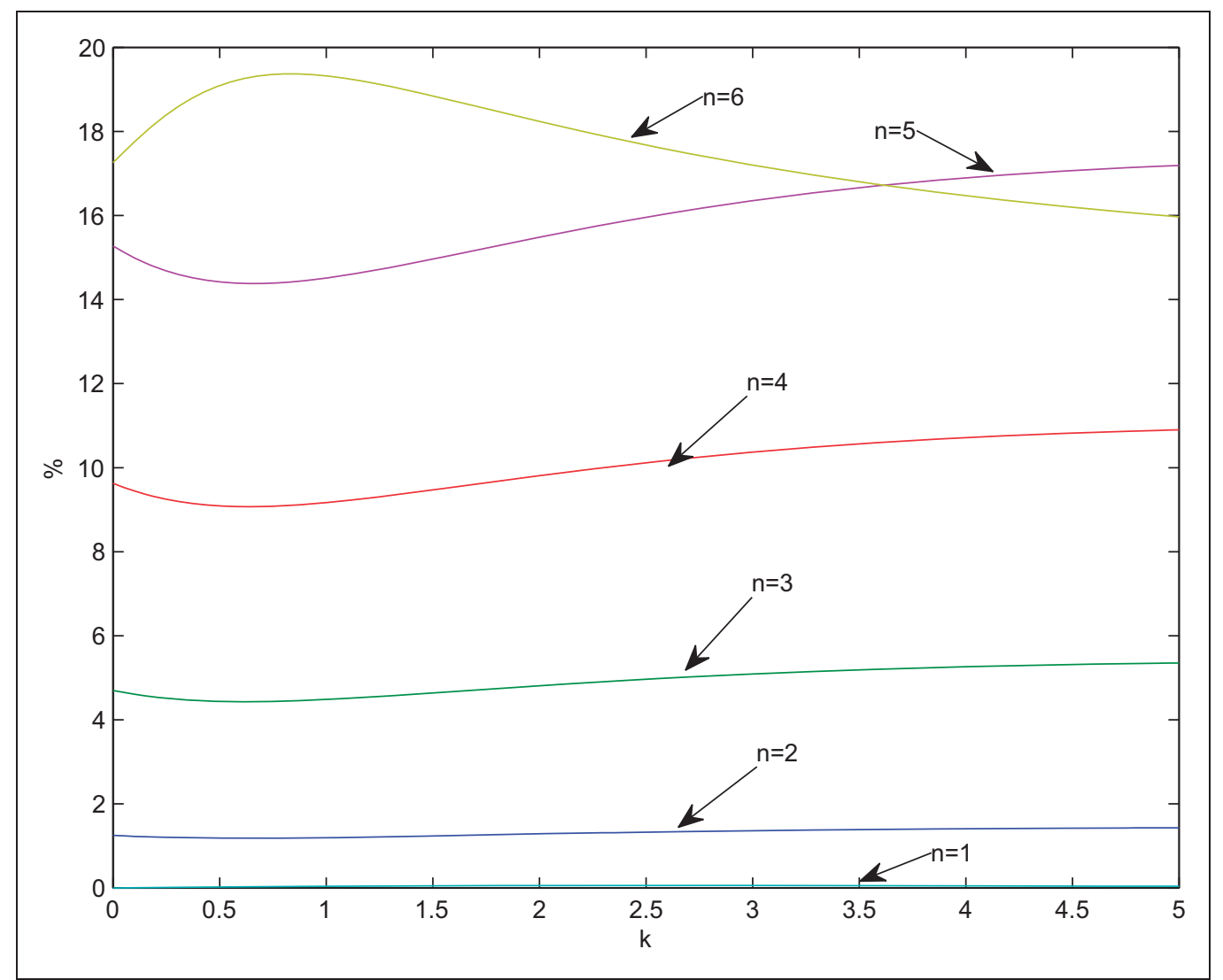

Figure 3. Frequency error for $\frac{L}{h}=10$.

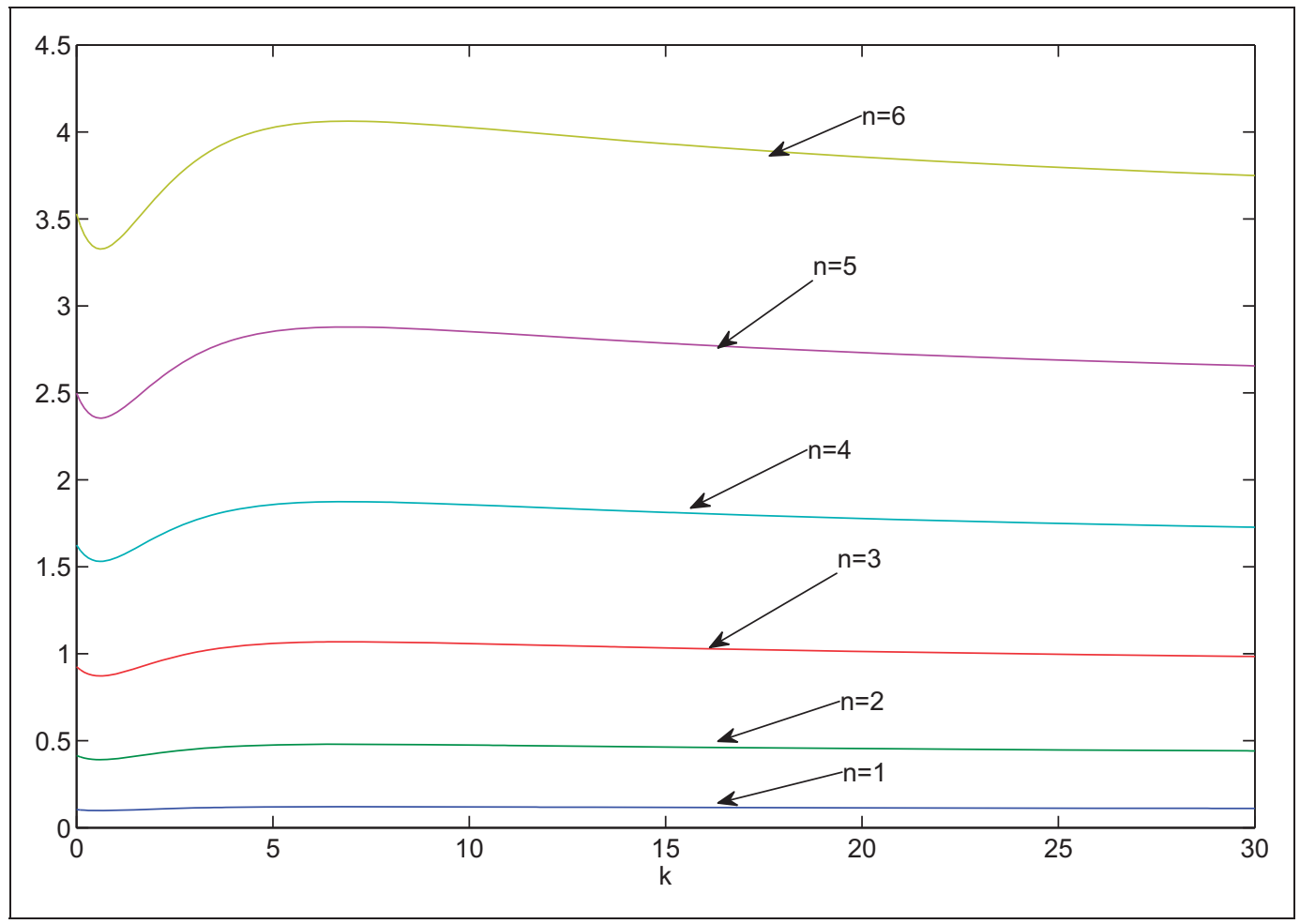

Figure 4. Frequency error for $\frac{L}{h}=35$. 


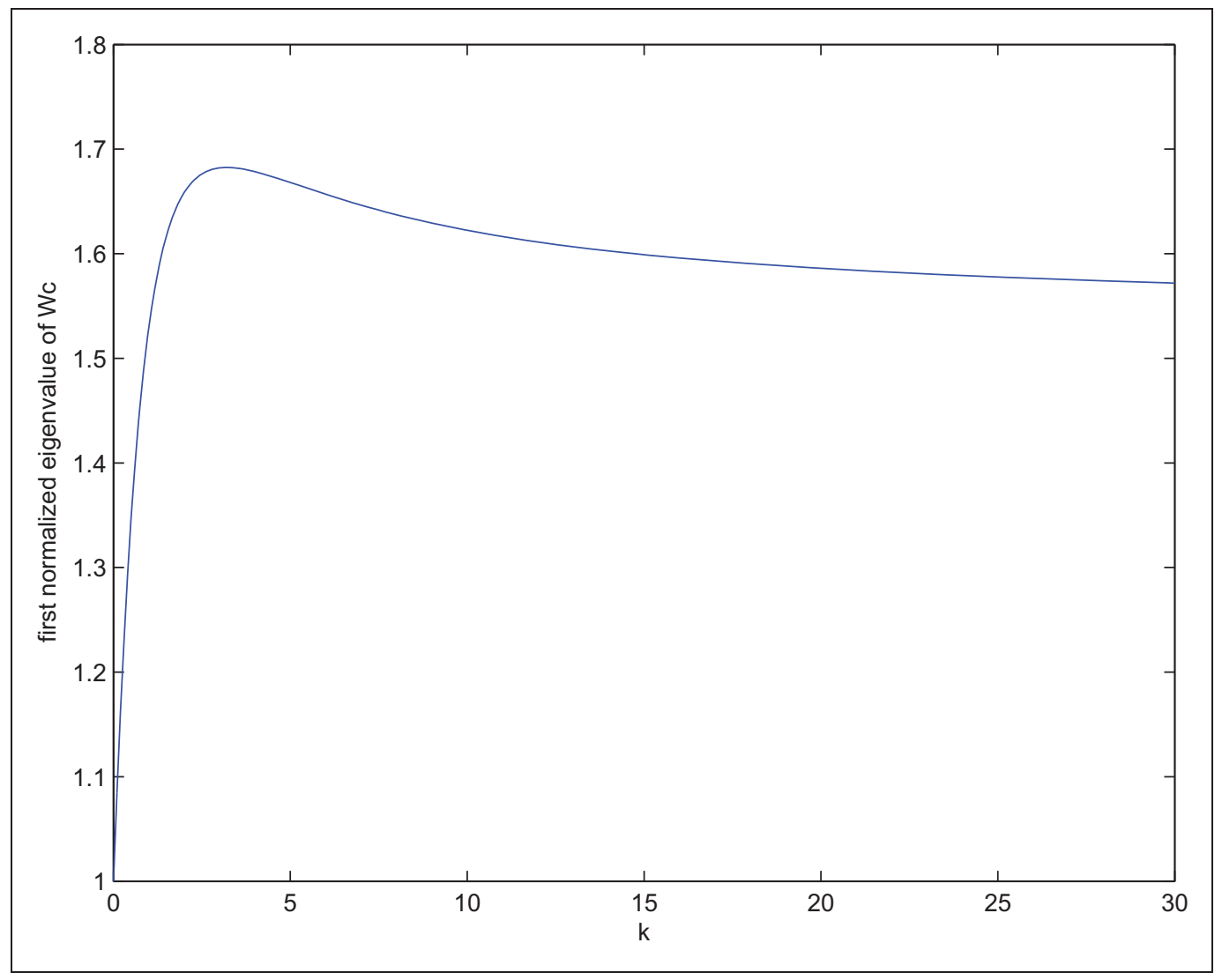

Figure 5. First normalized eigenvalue of $W_{c}$.

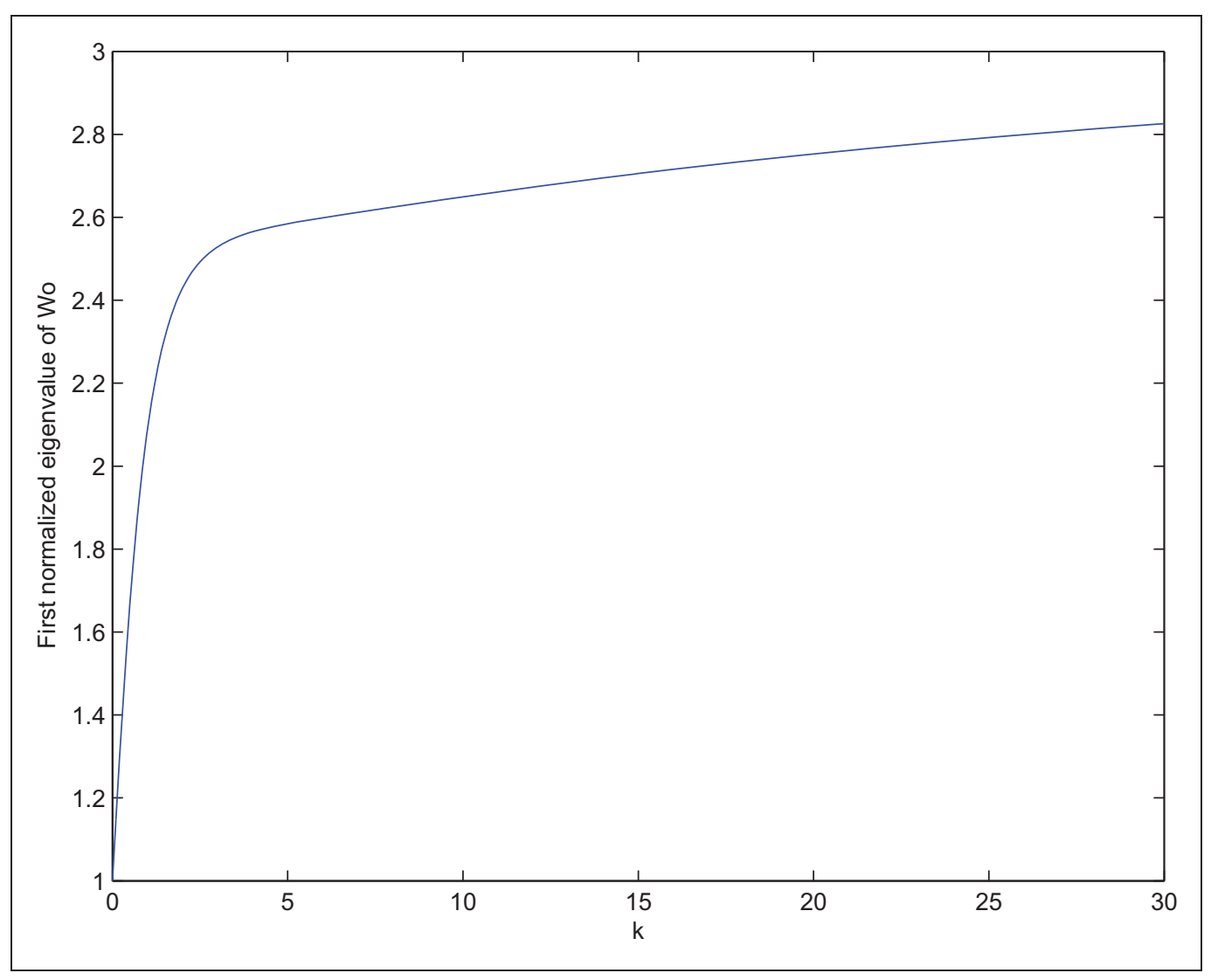

Figure 6. First normalized eigenvalue of $W_{0}$. 


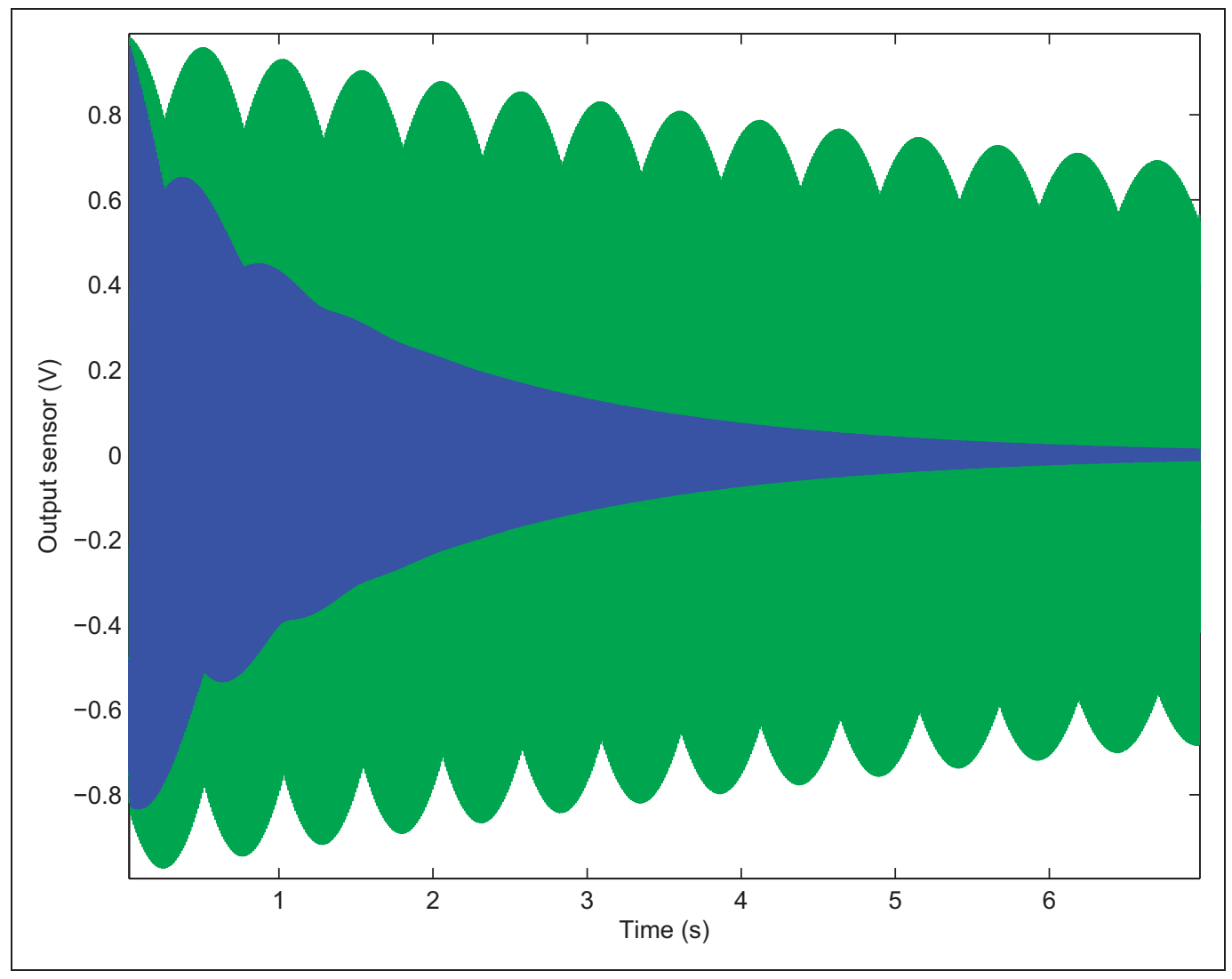

Figure 7. Test I: The sensor output for the open loop (green line) and closed loop (blue line).

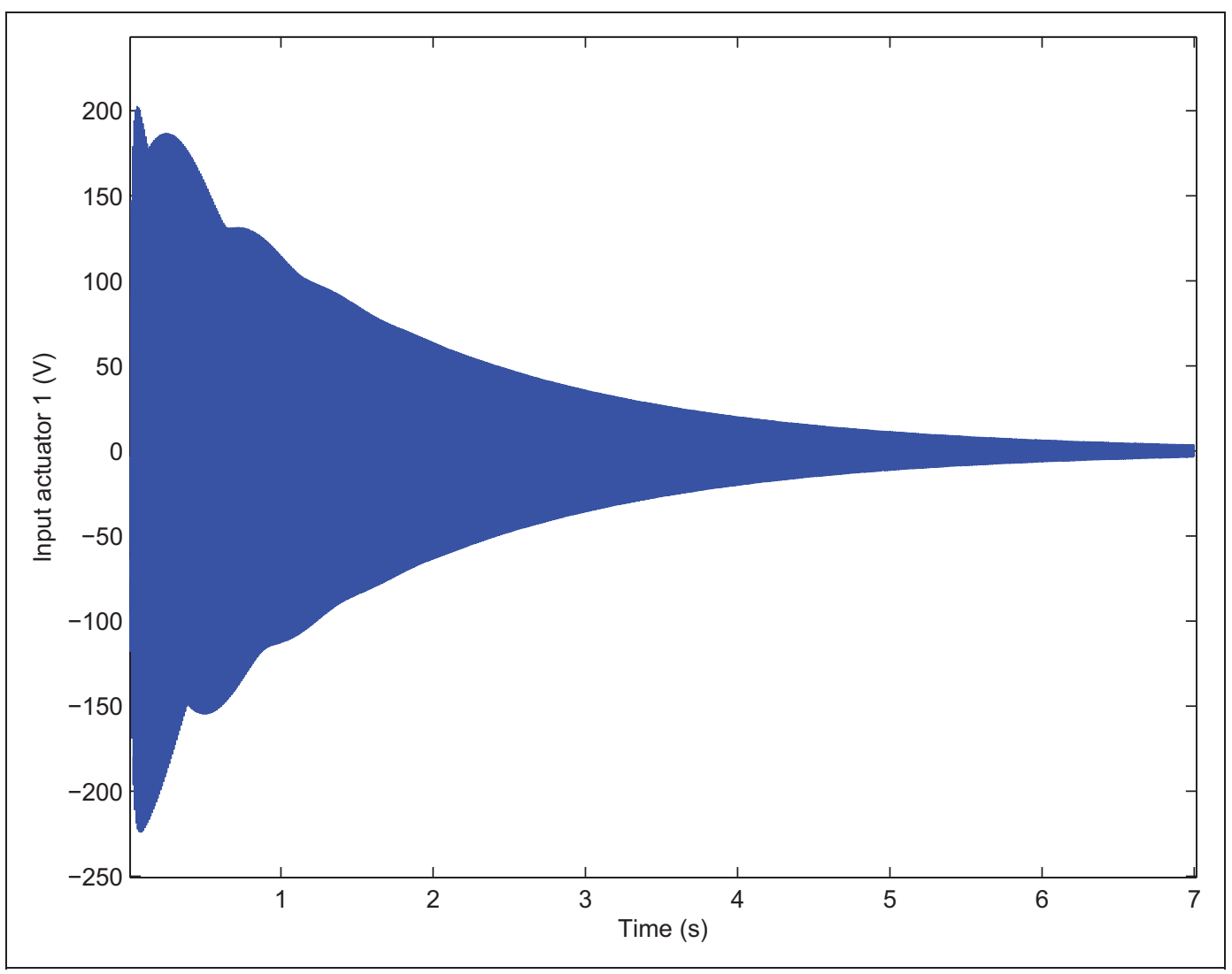

Figure 8. Test I: The actuator input $\mathrm{I}$. 


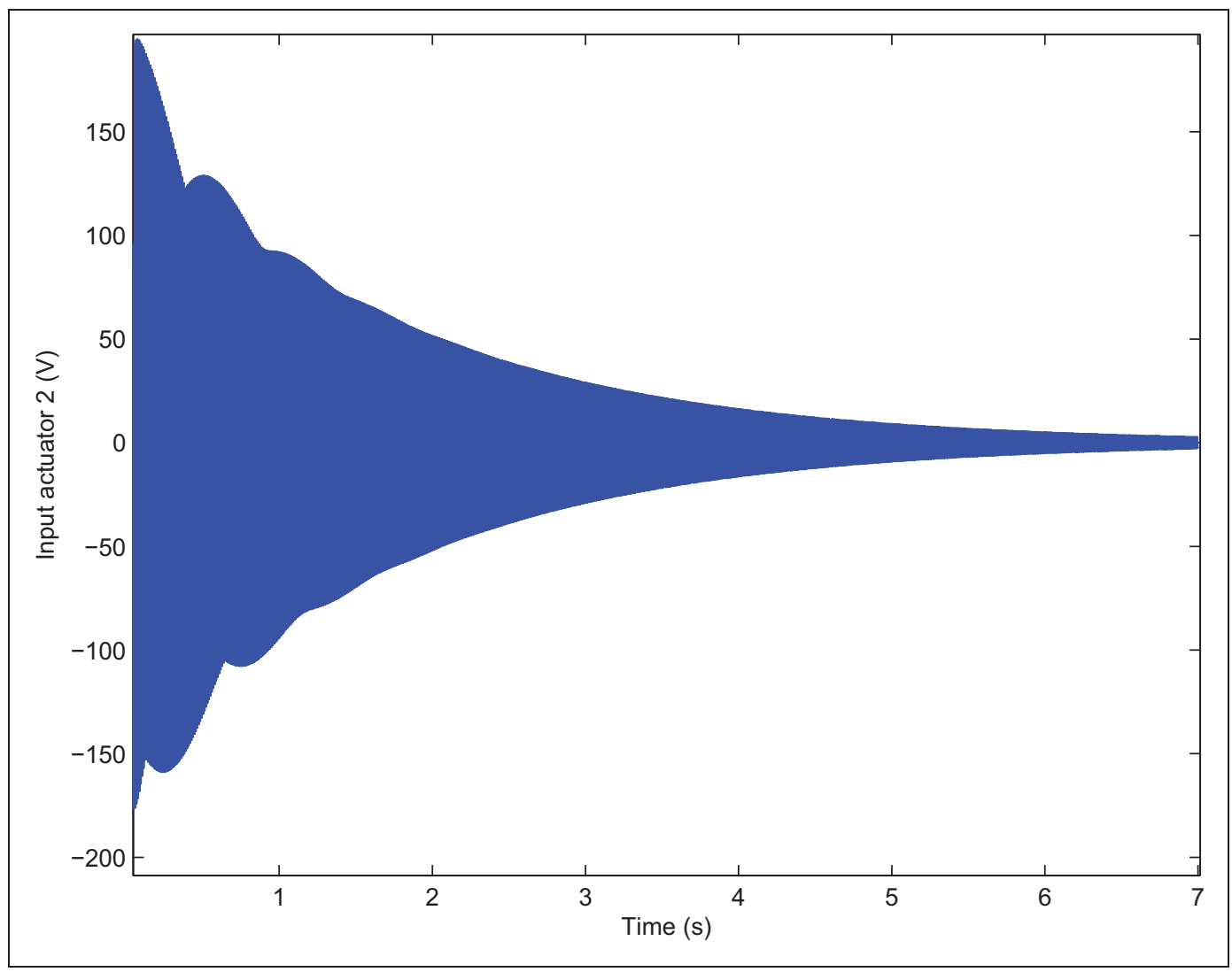

Figure 9. Test I: The actuator input 2.

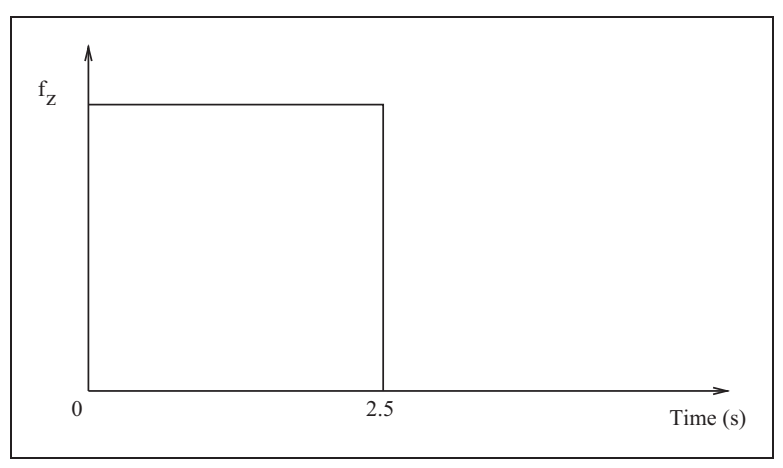

Figure 10. Test 2: Step load.

by one actuator located at $a_{1}=0.145 \mathrm{~m}$. The sensor output in open and closed loops, and the actuator input are presented in Figures 11 and 12 . The effectiveness of the active control again is shown.

4.3.3. Harmonic load test with one actuator. The structure is now subjected to a persistent harmonic load applied at $[L / 3, L / 3+L / 4]$ which equals $5.10^{4} \sin (754 t)$.
It is controlled by one actuator located at $a_{1}=0.145 \mathrm{~m}$. Results are plotted in Figures 13 and 14 and vibrations are controlled in less than $12 \mathrm{~s}$.

\subsection{Uncertainty in the volume fraction index $\mathrm{k}$ value}

Previous simulations present the efficiency of the active set up to control vibrations of FGM beam in the case $k=5$. Nevertheless, $k$ is an empirical parameter and its value is not necessarily accurate. Moreover the study of the $k$ influence on the controllability and observability index shows that for $k \in[0,5]$ these quantities vary strongly. Consequently, it is interesting to study the impact of uncertainty in the $k$ value on the vibrations and active control.

In this subsection, an error of $5 \%$ is considered for the parameter $k$. Figure 15 shows, for several values of $k$, the variation of the relative error of $E(z, k)$, in relation of $z$, defined by:

$$
\frac{E(z, k)-E(z, k(1+0.05))}{E(z, k(1+0.05))}
$$




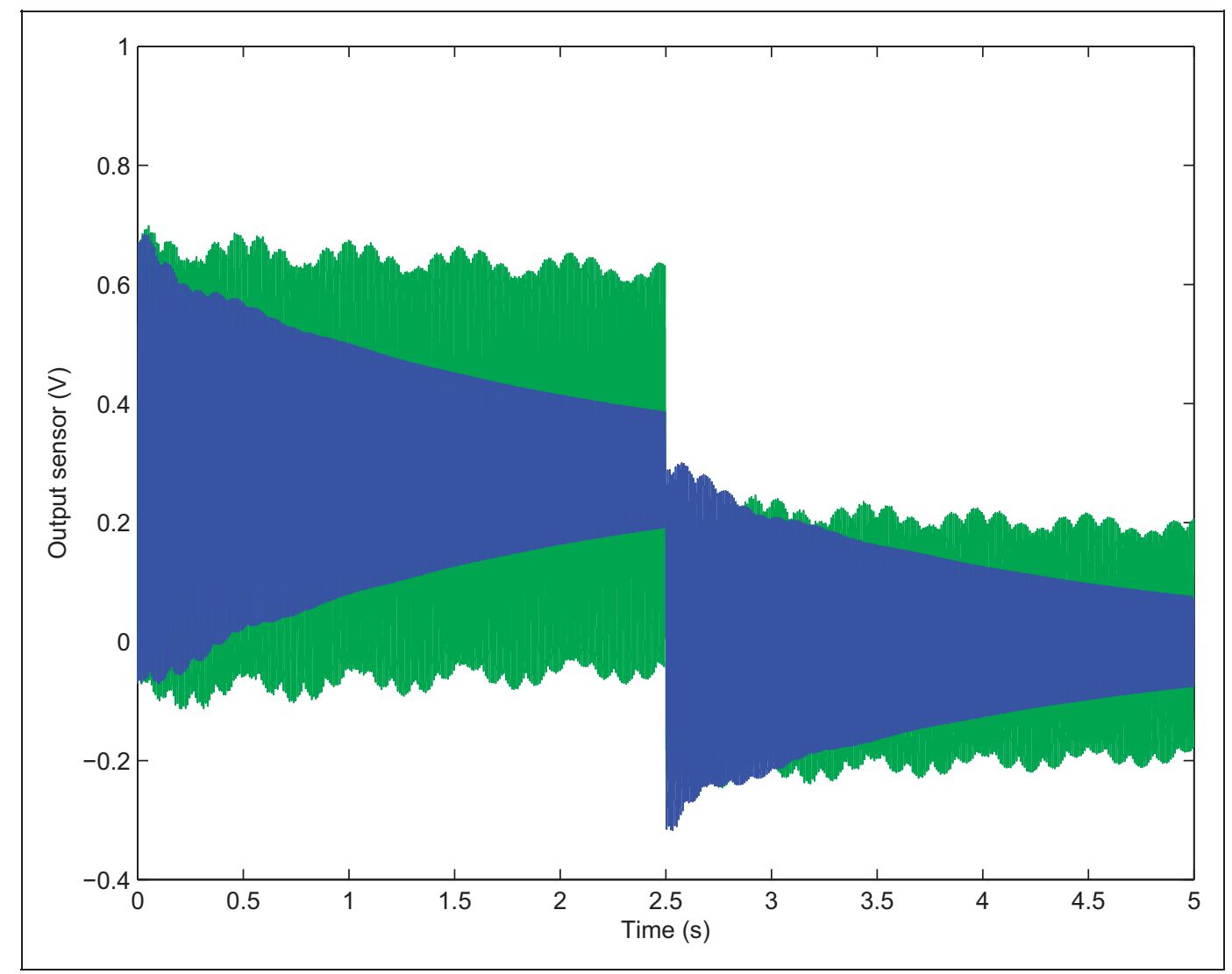

Figure II. Test 2: The sensor output for the open loop (green line) and closed loop (blue line).

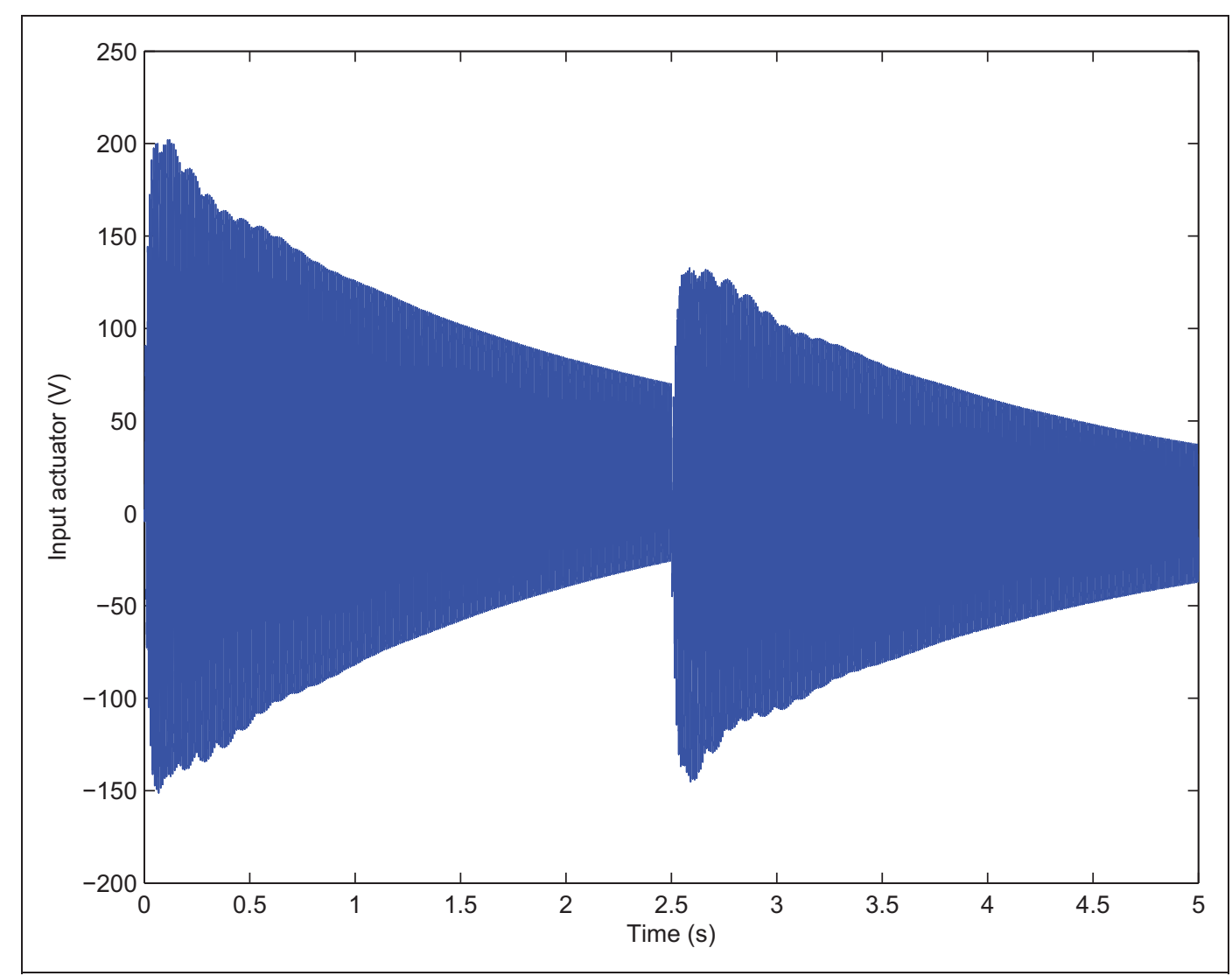

Figure 12. Test 2: The actuator input. 


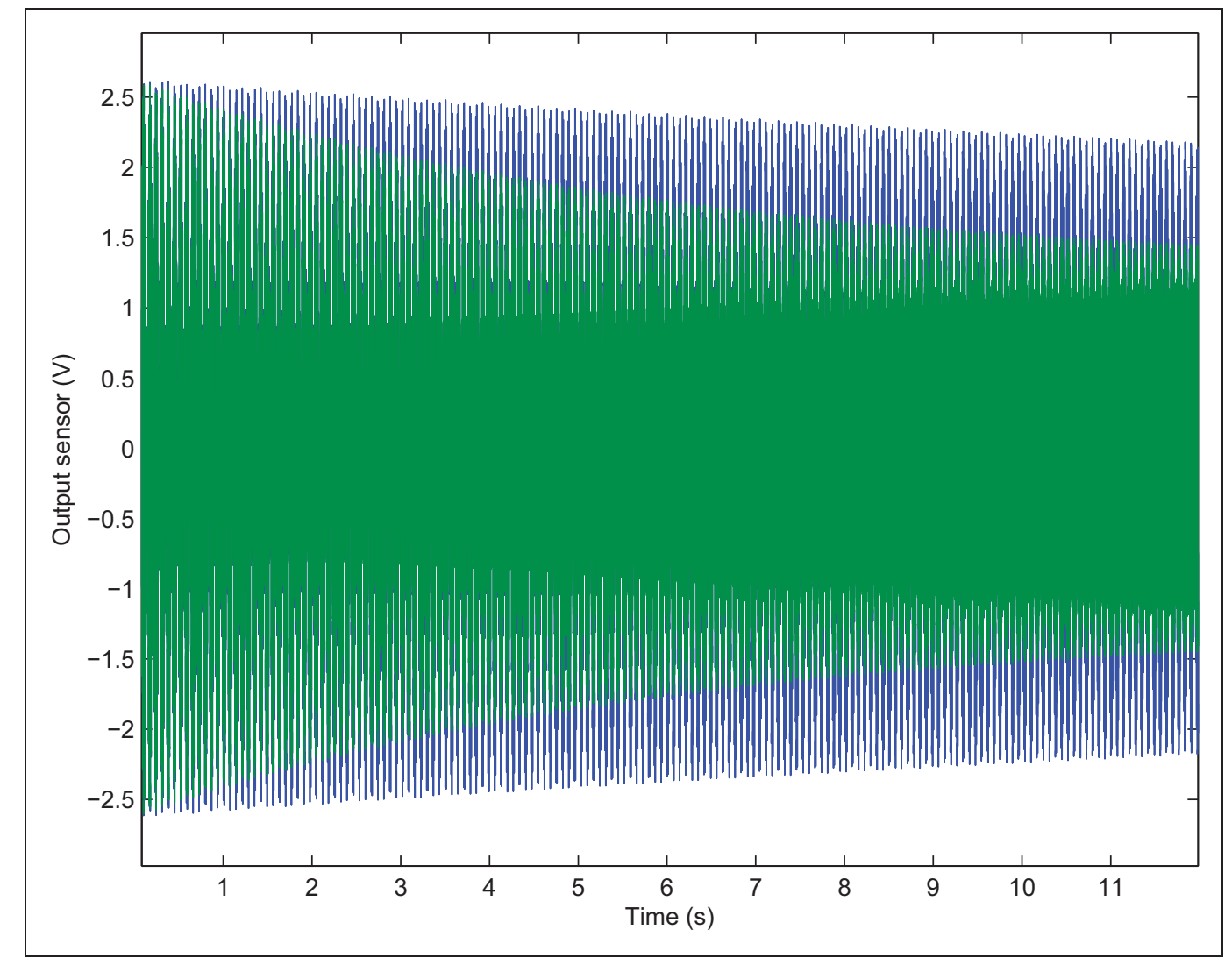

Figure 13. Test 3: The sensor output for the open loop (blue line) and closed loop (green line).

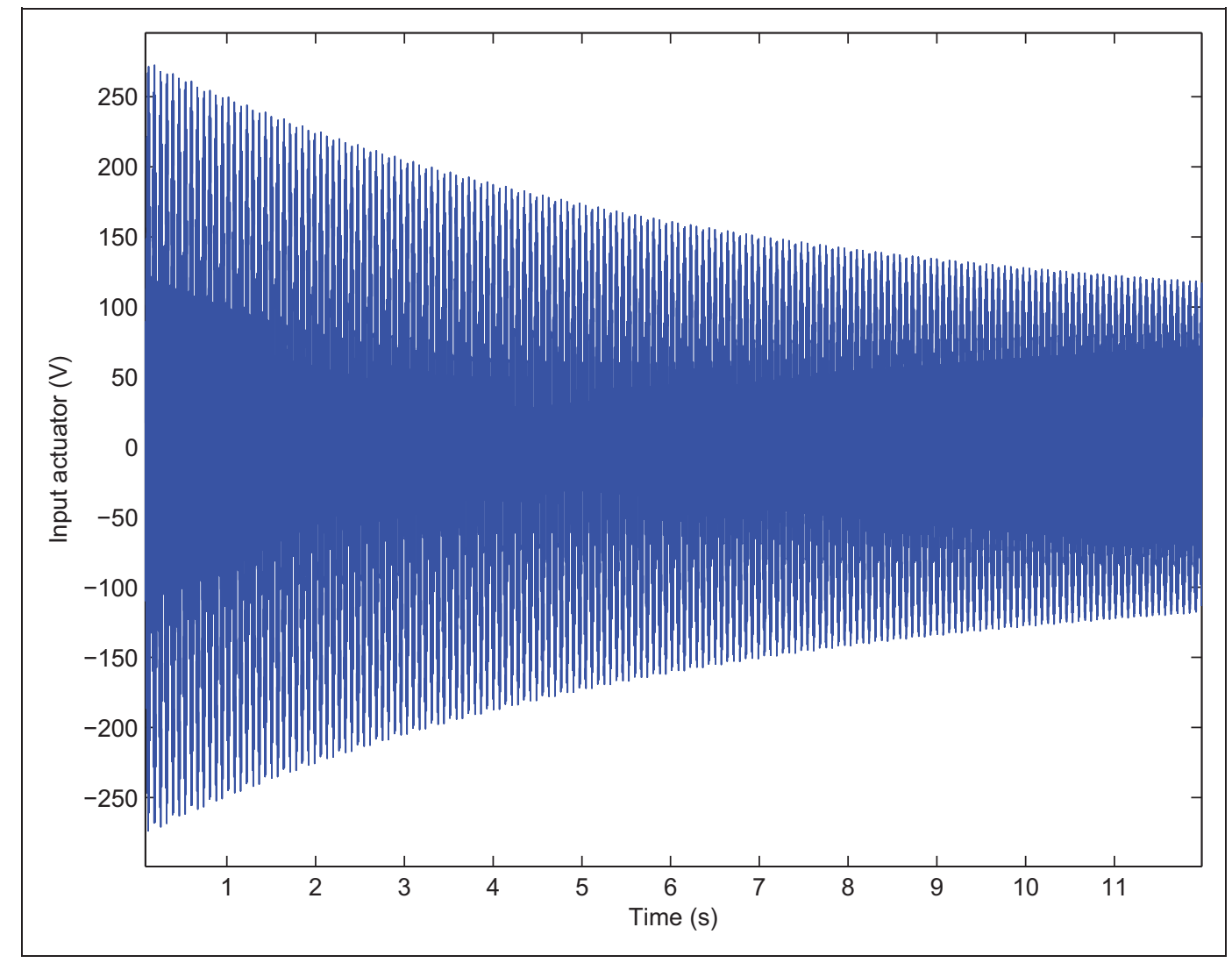

Figure 14. Test 3: The actuator input. 


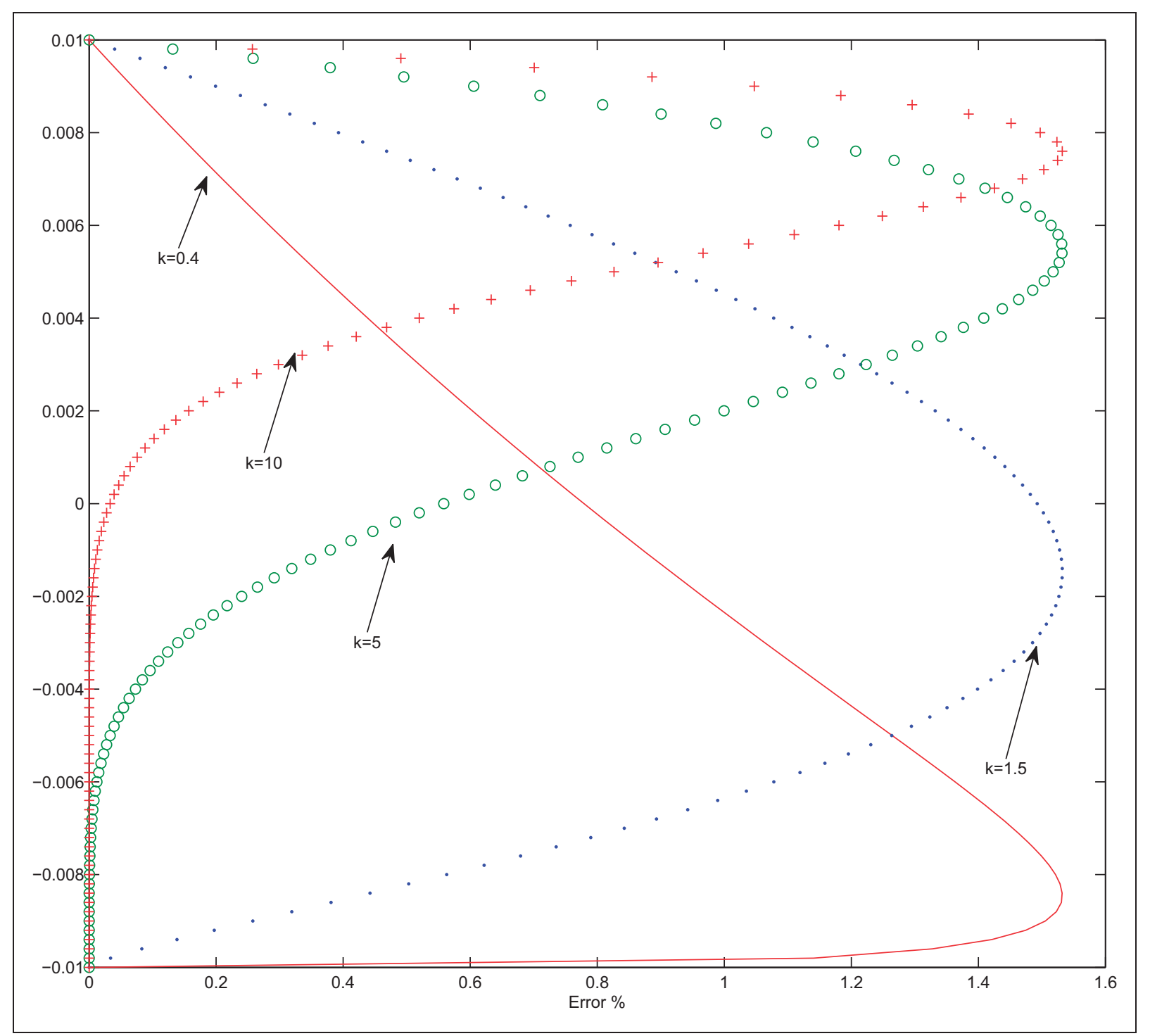

Figure I5. The relative error of $E(z, k)$.

For small values of $k(0.4 \leq k \leq 5)$, the variation of the error is present throughout the thickness of the beam, while it is more concentrated in the case of $k \geq 10$.

The relative errors for the six eigenfrequencies, in relation with $k$, are given figure (16). All curves are similar: the errors decrease for $k \in[0 ; 4]$ to a stable value. Consequently, for small values of $k$, an uncertainty in the $k$ value allows the dynamics of the structure to change and can affect the active control performances.

In the next simulations, we show two tests where results of vibration are strongly influenced by the parameter $k$. The actuator and the sensor are again located in $a_{1}=0.145 \mathrm{~m}$ and $c_{1}=0.495 \mathrm{~m}$. $k$ equals 0.475 and an error of $5 \%$ is added. The two tests are the following:

1. The beam is subjected to a step load $5.10^{4} \mathrm{~N}$ at the location $[L / 3, L / 3+L / 4]$ for $1 \mathrm{~s}$. Results are plotted Figures $17-19$.
2. The beam is subjected to persistent harmonic load applied at $[L / 3, L / 3+L / 4]$ and equals $10^{3} \sin (750 t)$. The load frequency is nearly the first eigenfrequency of the FGM beam. The input actuator and output sensor are plotted in Figures 20 and 21.

In each test, two simulations are considered in closed loop:

- Simulation without an error in the $k$ value: $k=0.5$ and the LQR regulator and observer are developed for this $k$ value,

- Simulation with a $5 \%$ error in the $k$ value: $k=0.475$ but we apply a LQR regulator and observer developed for $k=0.5$.

In the first test, for the open loop case, the difference between the two simulations is very important. This is 


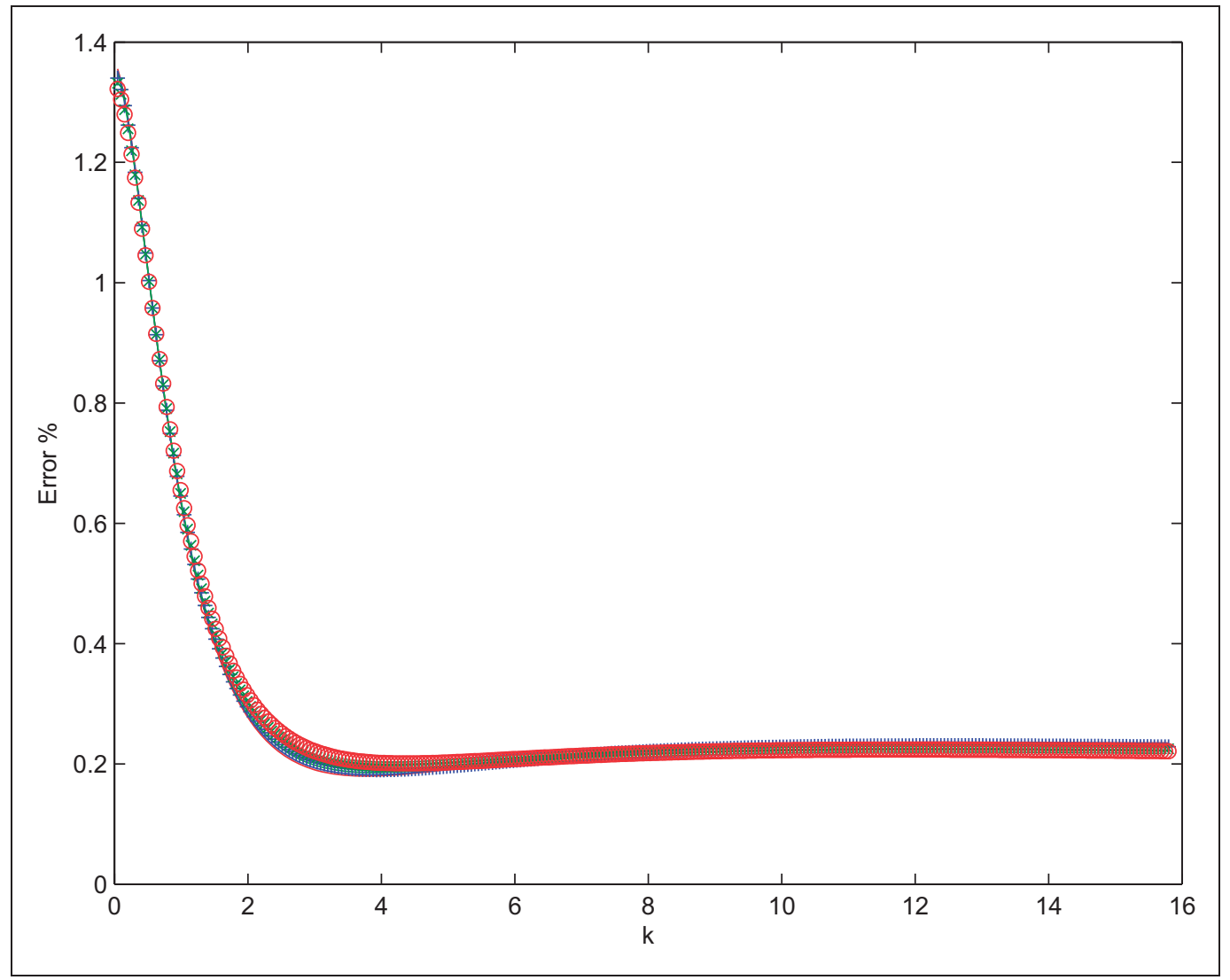

Figure 16. The relative error of the six first frequencies.

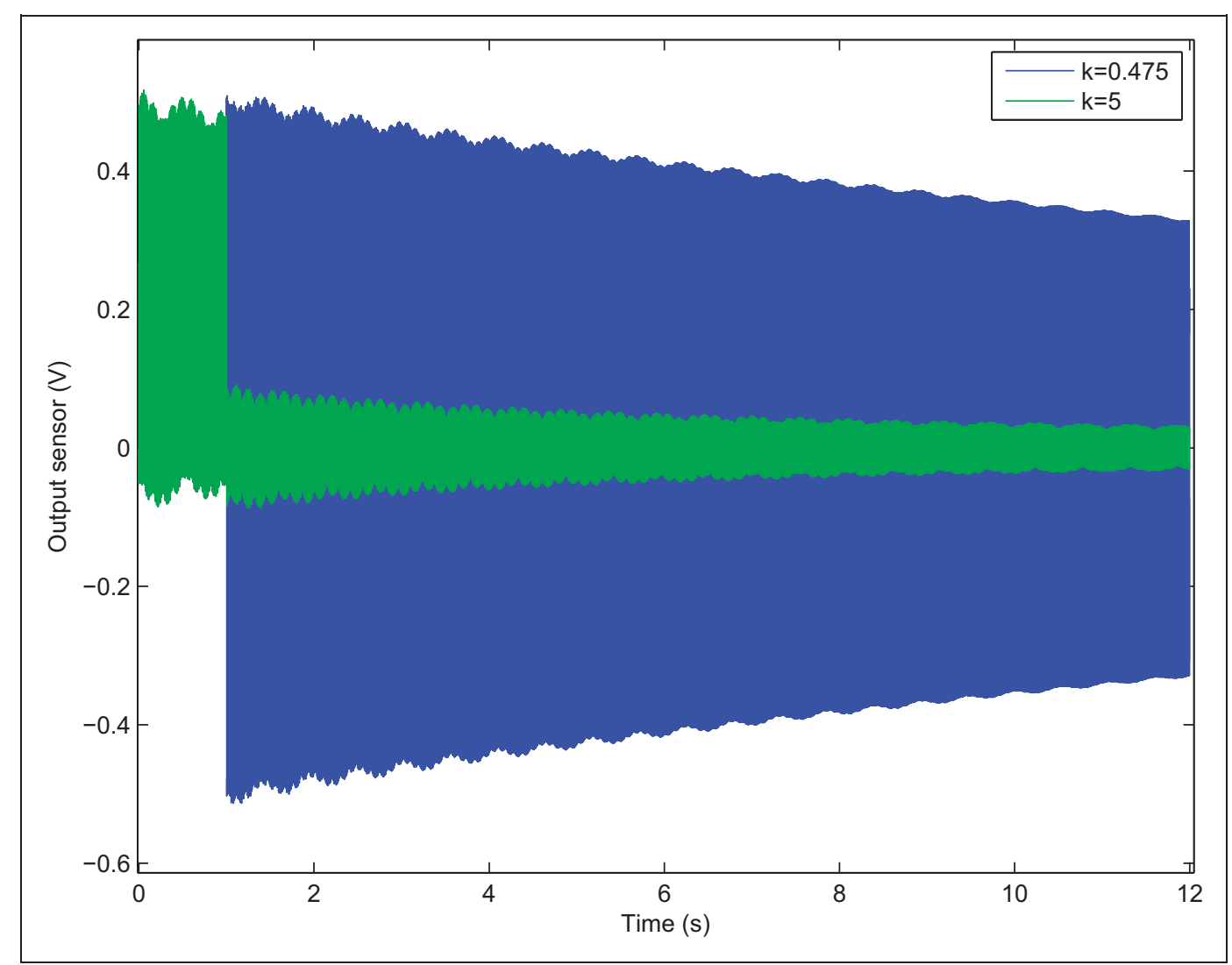

Figure 17. The sensor output for the step load in open loop. 


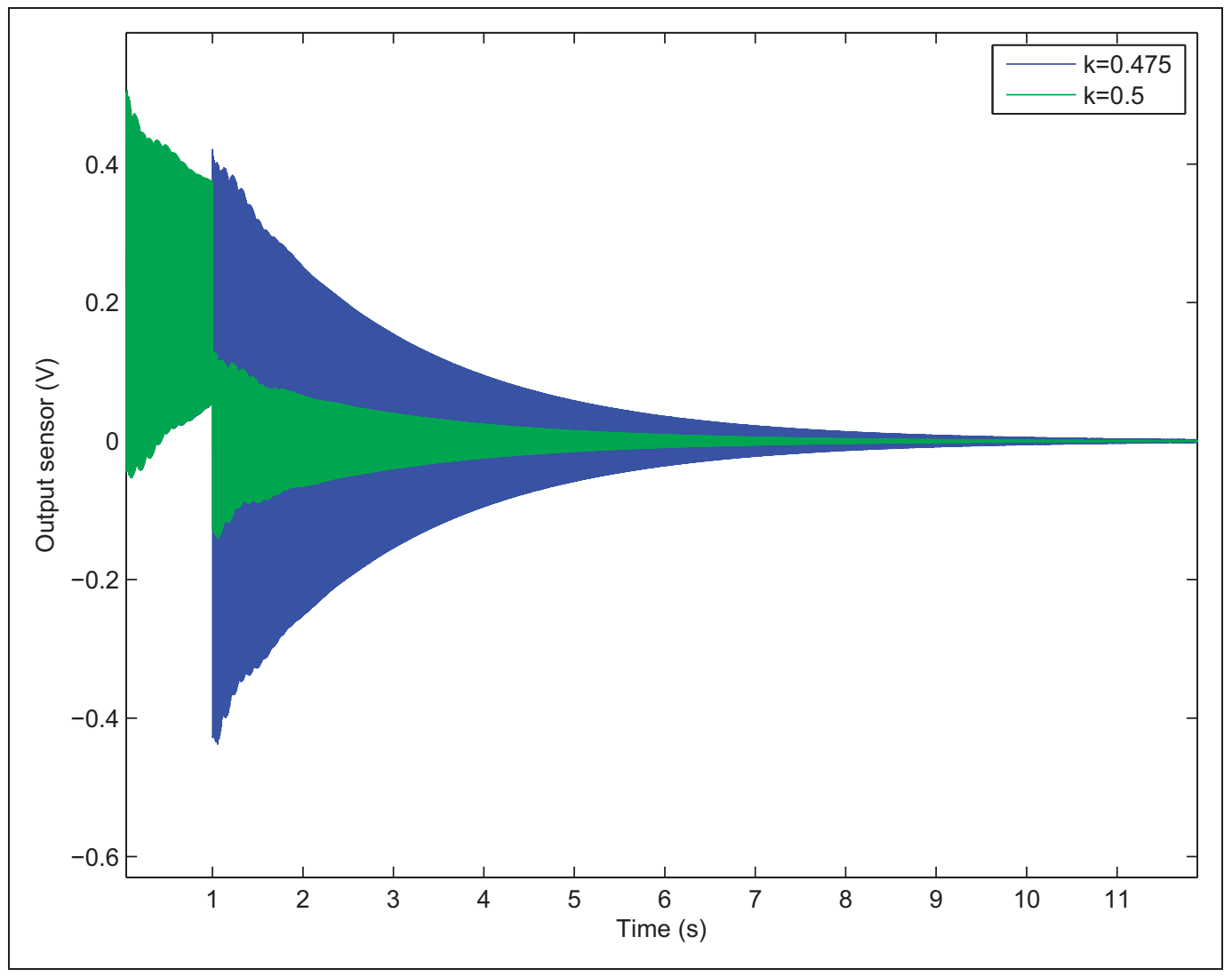

Figure 18. The sensor output for the step load in closed loop.

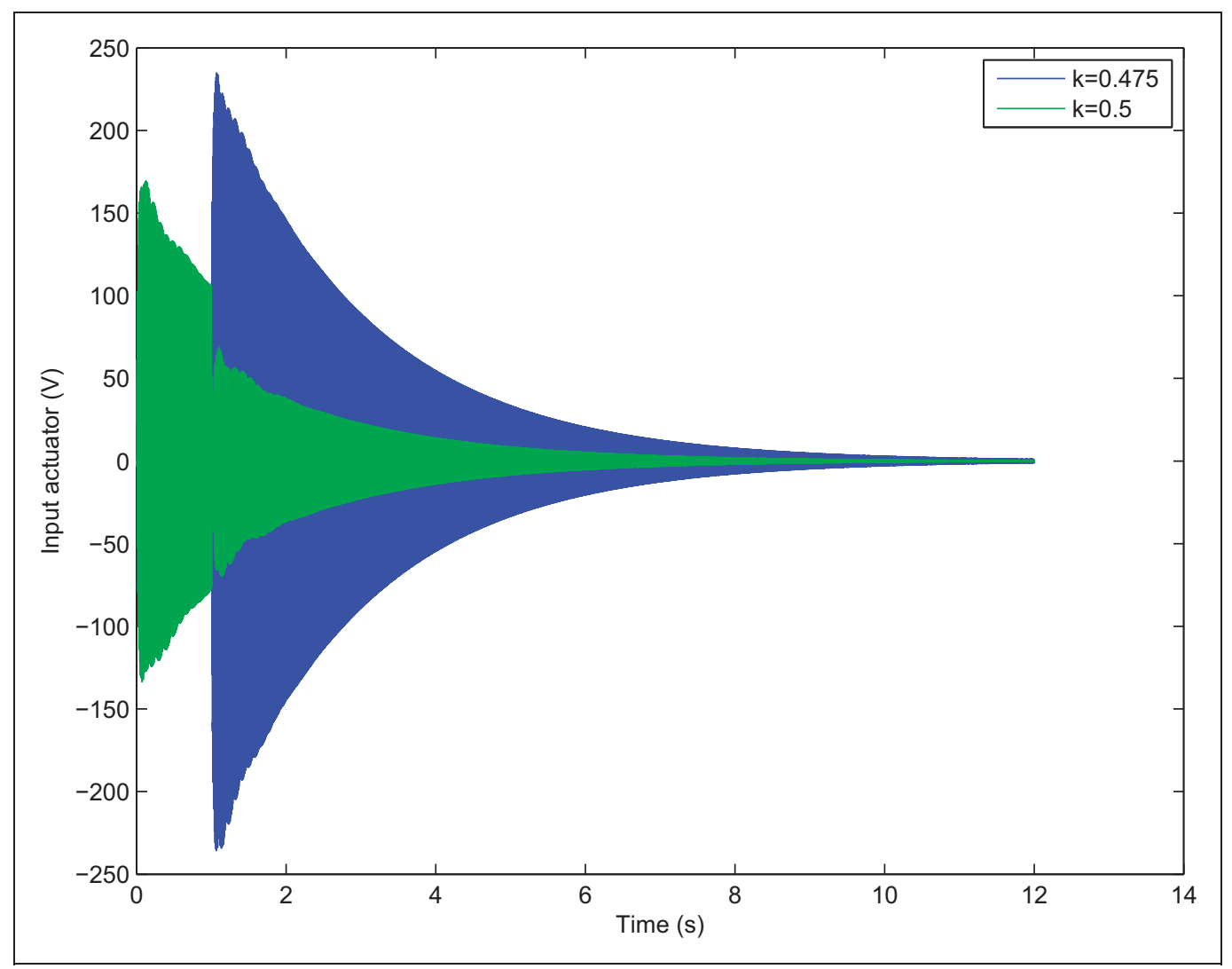

Figure 19. The actuator input for the step load. 


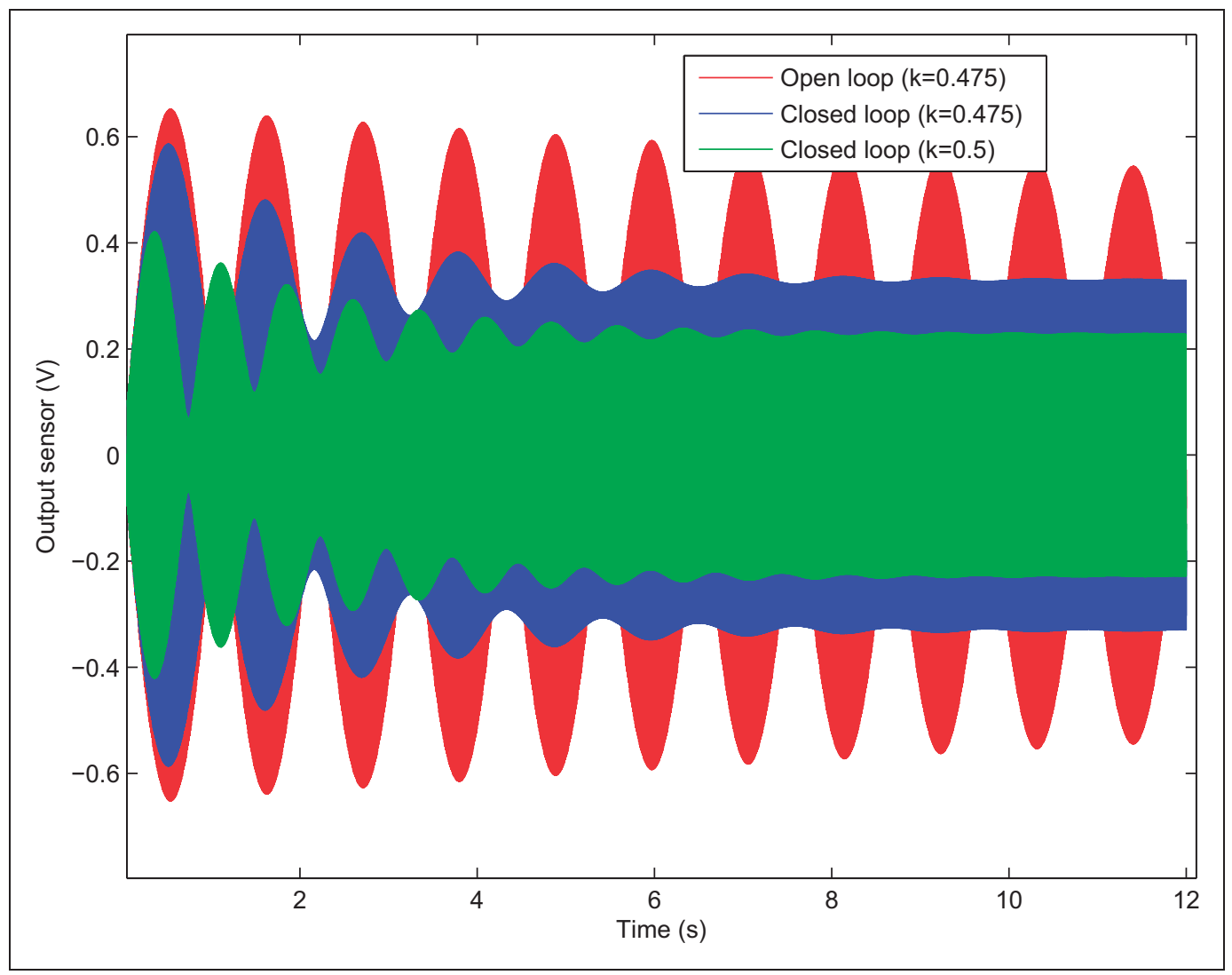

Figure 20. The sensor output for the sinus load.

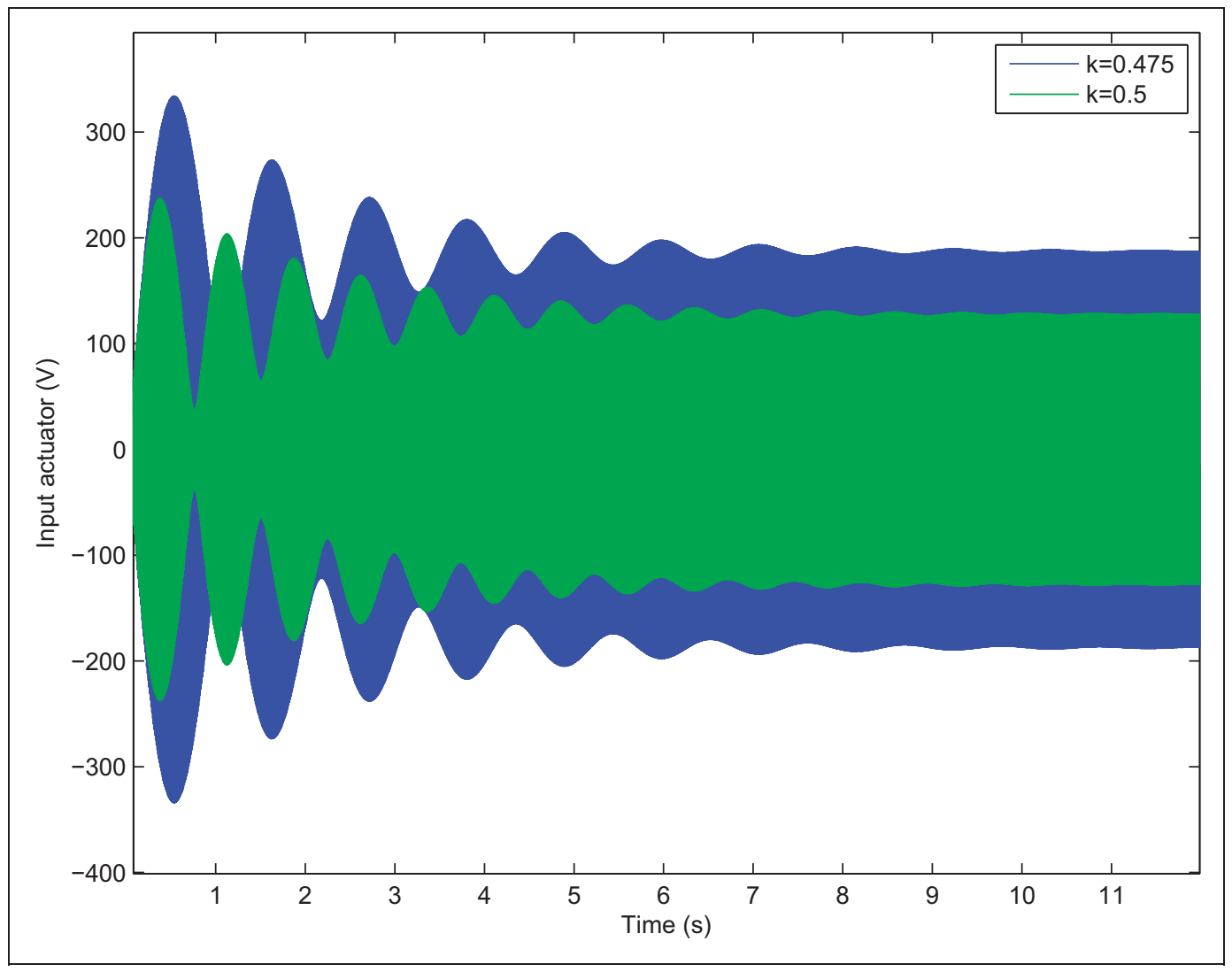

Figure 21. The actuator input for the sinus load. 
due to the load which is stopped suddenly. For the two tests, the regulator (developed for $k=0.5$ ) sufficiently controls the vibrations for $k=0.475$ : the LQR control is robust. Nevertheless, the maximal values of the input actuator are very high. The maximal admissible value of the piezoelectric patch can easily be exceeded.

\section{Conclusion}

This paper deals with the implementation of the active control tools for FGM beams control vibrations, equipped with piezoelectric actuators and sensors. Different from other investigations, the main steps to set up the active control are considered. First, an analytical formulation, based on a trigonometric shear deformation theory has been used to accurately model the dynamic behavior of the structure, and to deduce a state space control system. Then, the actuators' and sensors' locations are defined using an optimization procedure to well observe and control the first eigenmodes. Finally, an efficient LQR control law including a state observer has been computed.

The simulations clearly show that:

- The volume fraction index $k$ influences the controllability and observability of the system.

- When the actuators' and sensors' locations are defined in order to maximize the observability and controllability of each mode in the same range, $k$ does not influence the optimal locations.

- The active control of FGM, equipped with patches only bonded on the bottom of the beam, using the LQR control law, is very efficient, for several kinds of excitations, compared to the open loop simulations.

- Even if there is an error in the volume fraction index, the LQR law is robust and sufficiently controls the vibration. But a kind of uncertainty can induce values too high for the input actuators. In this way, the active control system could be damaged.

This work is a first step towards the active vibration control of FGM, as we have considered the FGM beam in the thickness direction here. In future studies, the described set-up of active control could be applied to an axially FGM beam and also to more realistic structures like FGM plates and shells equipped with several piezoelectric actuators and sensors.

\section{Funding}

This research received no specific grant from any funding agency in the public, commercial, or not-for-profit sectors.

\section{References}

Alshorbagy AE, Eltaher MA and Mahmoud FF (2011) Free vibration characteristics of a functionally graded beam by finite element method. Applied Mathematical Modelling 35: 412-425.

Aminbgahai M, Murin J and Hutis V (2012) Modal analysis of the FGM-beams with continuous transversal symmetric and longitudinal variation of material properties with effect of large axial force. Engineering Structures 34: 314-329.

Ang KK and Wang SY (2002) Weighted energy linear quadratic regulator vibration control of piezoelectric composite plates. Smart Materials and Structures 11: 98-106.

Atmane HA, Tounsi A, Meftah SA and Belhadj HA (2010) Free vibration behavior of exponential functionally graded beams with varying cross-section. Journal of Vibration and Control 17: 311-318.

Aydogdu M and Taskin V (2006) Free vibration of functionally graded beams with simply supported edges. Materials and Design 28: 1651-1656.

Balamurugan V and Narayanan S (2001) Shell finite element for smart piezoelectric composite plate/shell structures and its application to the study of active vibration control. Finite Elements in Analysis and Design 37: 713-738.

Benjeddou A (2000) Advances in piezoelectric finite element modeling of adaptive structural elements: A survey. Computers and Structures 76: 347-363.

Bruant I, Coffignal G, Léné F and Vergé M (2001) Active control of beam structures with piezoelectric actuators and sensors: Modeling and simulation. Smart Materials and Structures 10: 404-408.

Bruant I, Pablo F and Polit O (2008) Active control of laminated plates using a piezoelectric finite element. Mechanics of Advanced Materials and Structures 15: 276-290.

Bruant I, Gallimard L and Nikoukar S (2010) Optimal piezoelectric actuator and sensor location for active vibration control, using genetic algorithm. Journal of Sound and Vibration 329: 1615-1635.

Chomette B, Chesné S, Rémond D and Gaudiller L (2008) Semi adaptive modal control of on-board electronic boards using an identification method. Smart Materials and Structures 17: 065019.

Doroushi A, Eslami MR and Komeili A (2011) Vibration analysis and transient response of an FGPM beam under thermo-electro-mechanical loads using higher-order shear deformation theory. Journal of Intelligent Material Systems and Structures 22: 231-243.

Fakhari V and Ohadi A (2010) Nonlinear vibration control of functionally graded plate with piezoelectric layers in thermal environment. Journal of Vibration and Control 17: 448-469.

Fu Y, Wang J and Mao Y (2013) Nonlinear vibration and active control of functionally graded beams with piezoelectric sensors and actuators. Journal of Intelligent Material Systems and Structures 22: 2093-2102.

Gaudiller L and Der Hagopian J (1996) Active control of flexible structures using a minimum number of components. Journal of Sound and Vibration 193: 713-741. 
Gharib A, Salehi M and Fazeli S (2008) Deflection control of functionally graded material beams with bonded piezoelectric sensors and actuators. Materials Science and Engineering $A$ 498: 110-114.

Giunta G, Crisafulli D, Belouettar S and Carrera E (2011) Hierarchical theories for the free vibration analysis of functionally graded beams. Composite Structures 94: $68-74$.

Hac A and Liu L (1993) Sensor and actuator location in motion control of flexible structures. Journal of Sound and Vibration 167: 239-261.

He XQ, Ng TY, Sivashanker S and Liew KM (2001) Active control of FGM plates with integrated piezoelectric sensors and actuators. International Journal of Solids and Structures 38: 1641-1655.

Jha AK and Inman DJ (2003) Optimal sizes and placements of piezoelectric actuators and sensors for an inflated torus. Journal of Intelligent Material Systems and Structures 14: $563-576$

Jha DK, Kant T and Singh RK (2013) A critical review of recent research on functionally graded plates. Composite Structures 96: 833-849.

Kailath T (1980) Linear Systems. Englewood Cliffs, NJ: Prentice Hall.

Kapuria S, Bhattacharyya M and Kumar AN (2007) Bending and free vibration response of layered functionally graded beams: A theoretical model and its experimental validation. Composite Structures 82: 390-402.

Kargarnovin MH, Najafizadeh MM and Viliani NS (2007) Vibration control of functionally graded material plate patched with piezoelectric actuators and sensors under a constant electric charge. Smart Materials and Structures 16: $1252-1259$.

Kondoh S, Yatomi C and Inoue K (1990) The positioning of sensors and actuators in the vibration control of flexible systems. JSME International Journal 33: 145-152.

Kusculuoglu ZK and Royston TJ (2005) Finite element formulation for composite plates with piezoceramic layers for optimal vibration control applications. Smart Materials and Structures 14: 1139-1153.

Li XF (2008) A unified approach for analyzing static and dynamic behaviors of functionally graded Timoshenko and Euler-Bernoulli beams. Journal of Sound and Vibration 318: 1210-1229.

Liew KM, Sivashanker S, He XQ and Ng TY (2003) The modeling and design of smart structures using functionally graded materials and piezoelectrical sensor/actuator patches. Smart Materials and Structures 12: 647-655.

Mirzaeifar R, Bahai H and Shahab S (2008) Active control of natural frequencies of FGM plates by piezoelectric sensor/ actuator pairs. Smart Materials and Structures 17: 045003.

Narayanan S and Balamurugan V (2003) Finite element modelling of piezolaminated smart structures for active vibration control with distributed sensors and actuators. Journal of Sound and Vibration 262: 529-562.

Narayanan S and Balamurugan V (2010) Functionally graded shells with distributed piezoelectric sensors and actuators for active vibration control. IUTAM symposium on multi functional material structures and systems, Bangalore, India, 10-12 December 2008, pp.3-13. Springer.
Nestorovic T and Trajkov M (2010) Active control of smart structures-An overall approach. Facta Universitatis: Architecture and Civil Engineering 8: 35-44.

Polit O and Touratier M (2000) High-order triangular sandwich plate finite element for linear and non-linear analyses. Computer Methods in Applied Mechanics and Engineering 185: 305-324.

Preumont A (1999) Vibration Control of Active Structures: An Introduction. Dordrecht, The Netherlands: Kluwer Academic Publishers.

Reddy JN (2004) Mechanics of Laminated Composite Plates and Shells: Theory and Analysis. Boca Raton, FL: CRC Press.

Sheng GG and Wang X (2009) Active control of functionally graded laminated cylindrical shells. Composite Structures 90: 448-457.

Simsek M (2010) Fudamental frequency analysis of functionally graded beams by using different higher-order beam theories. Nuclear Engineering and Design 240: 697-705.

Simsek M and Kocaturk T (2009) Free and forced vibration of a functionally graded beam subjected to a concentrated moving harmonic load. Composite Structures 90: 465-473.

Sina SA, Navazi HM and Haddadpour H (2009) An analytical method for free vibration analysis of functionally graded beams. Materials and Design 30: 741-747.

Xiang HJ and Yang J (2008) Free and forced vibration of a laminated FGM Timoshenko beam of variable thickness under heat conduction. Composites: Part B 39: 292-303.

Yao RX and Shi ZF (2011) Steady-state forced vibration of functionally graded piezoelectric beams. Journal of Intelligent Material Systems and Structures 22: 769-779.

Yiqi M and Yiming F (2010) Nonlinear dynamic response and active vibration control for piezoelectric functionally graded plate. Journal of Sound and Vibration 329: 2015-2028.

Zheng SJ, Dai F and Song Z (2009) Active control of piezothermoelastic FGM shells using integrated piezoelectric sensor/actuator layers. International Journal of Applied Electromagnetics and Mechanics 30: 107-124.

\section{Appendix A. Definition of the coefficients " $\langle\cdot\rangle$ "}

For a function $F(y, z)$ :

$$
\begin{aligned}
\langle E, F\rangle & =\int_{-h / 2}^{h / 2} \int_{-b / 2}^{b / 2} E(z, k) F(y, z) \mathrm{d} y \mathrm{~d} z \\
\langle m, F\rangle & =\int_{-h / 2}^{h / 2} \int_{-b / 2}^{b / 2} \rho(z, k) F(y, z) \mathrm{d} y \mathrm{~d} z \\
\left\langle e_{31}\right\rangle & =\int_{-h / 2}^{h / 2} \int_{-b / 2}^{b / 2} e_{31} \mathrm{~d} y \mathrm{~d} z \\
\left\langle e_{31}(f(z)-z)\right\rangle & =\int_{-h / 2}^{h / 2} \int_{-b / 2}^{b / 2}(f(z)-z) e_{31} \mathrm{~d} y \mathrm{~d} z
\end{aligned}
$$




$$
\begin{aligned}
\left\langle e_{31} f\right\rangle & =\int_{-h / 2}^{h / 2} \int_{-b / 2}^{b / 2} f(z) e_{31} \mathrm{~d} y \mathrm{~d} z \\
\left\langle G f^{\prime 2}\right\rangle & =\int_{-h / 2}^{h / 2} \int_{-b / 2}^{b / 2} \frac{E(z, k)}{2(1+v)}\left(f^{\prime}(z)\right)^{2} \mathrm{~d} y \mathrm{~d} z \\
\left\langle f_{z}\right\rangle & =\int_{-h / 2}^{h / 2} \int_{-b / 2}^{b / 2} f_{z}(y, z) \mathrm{d} y \mathrm{~d} z
\end{aligned}
$$

\section{Appendix B. Definition of matrices}

$M_{0}$ and $K_{0}$

$\mathbf{M}_{\mathbf{0}}$ and $\mathbf{K}_{\mathbf{0}}$ are $3 \times 3$ symmetric matrices, whose coefficients are:

$$
\begin{aligned}
& {\left[M_{0}\right]_{11}=\langle m, 1\rangle} \\
& {\left[M_{0}\right]_{12}=\frac{p \pi}{L}(-\langle m, z\rangle+\langle m, f\rangle)} \\
& {\left[M_{0}\right]_{13}=\langle m, f\rangle} \\
& {\left[M_{0}\right]_{22}=\langle m, 1\rangle-\left(\frac{p \pi}{L}\right)^{2}\left(-\left\langle m, z^{2}\right\rangle+2\langle m, z f\rangle-\left\langle m, f^{2}\right\rangle\right)} \\
& {\left[M_{0}\right]_{23}=-\frac{p \pi}{L}\left(-\langle m, z f\rangle+\left\langle m, f^{2}\right\rangle\right)} \\
& {\left[M_{0}\right]_{33}=\left\langle m, f^{2}\right\rangle}
\end{aligned}
$$

$$
\begin{aligned}
& {\left[K_{0}\right]_{11}=\left(\frac{p \pi}{L}\right)^{2}\langle E, 1\rangle} \\
& {\left[K_{0}\right]_{12}=\left(\frac{p \pi}{L}\right)^{3}(\langle E, f\rangle-\langle E, z\rangle)} \\
& {\left[K_{0}\right]_{13}=\left(\frac{p \pi}{L}\right)^{2}\langle E, f\rangle} \\
& {\left[K_{0}\right]_{22}=\left(\frac{p \pi}{L}\right)^{4}\left(\left\langle E, f^{2}\right\rangle+\left\langle E, z^{2}\right\rangle-2\langle E, f z\rangle\right)+\left(\frac{p \pi}{L}\right)^{2}\left\langle G f_{2}^{\prime}\right\rangle} \\
& {\left[K_{0}\right]_{23}=\left(\frac{p \pi}{L}\right)^{3}\left(-\langle E, z f\rangle+\left\langle E, f^{2}\right\rangle\right)+\frac{p \pi}{L}\left\langle G f_{2}^{\prime}\right\rangle} \\
& {\left[K_{0}\right]_{33}=\left(\frac{p \pi}{L}\right)^{2}\left\langle E, f^{2}\right\rangle+\left\langle G f_{2}^{\prime}\right\rangle}
\end{aligned}
$$

